\title{
THE DISTRIBUTION OF WASTED SPACES IN THE M/M/ $\infty$ QUEUE WITH RANKED SERVERS
}

\author{
EUNJU SOHN ${ }^{* * * * * * *}$ AND \\ CHARLES KNESSL, ${ }^{* * * * * * * *}$ University of Illinois at Chicago
}

\begin{abstract}
We consider the $\mathrm{M} / \mathrm{M} / \infty$ queue with $m$ primary servers and infinitely many secondary servers. All the servers are numbered and ordered. An arriving customer takes the lowest available server. We define the wasted spaces as the difference between the highest numbered occupied server and the total number of occupied servers. Letting $\rho=\lambda_{0} / \mu$ be the ratio of arrival to service rates, we study the probability distribution of the wasted spaces asymptotically for $\rho \rightarrow \infty$. We also give some numerical results and the tail behavior for $\rho=O(1)$.
\end{abstract}

Keywords: Ranked server; asymptotics; wasted spaces

2000 Mathematics Subject Classification: Primary 60K25

Secondary 34E10; 60J27

\section{Introduction}

We consider the following stochastic model. Near a restaurant there are $m$ primary parking spaces. They are numbered and ordered; the one with rank 1 is closest to the restaurant. Suppose that across the street there are a large number of additional spaces, which we take to be infinite. These are also numbered and ordered. We assume the following: (i) customers arrive according to a Poisson process with rate $\lambda_{0}$, (ii) the amount of time that a customer occupies a given parking space is exponentially distributed with mean $1 / \mu$, and (iii) each arriving car parks in the lowest-numbered available space.

This model has many applications, including dynamic storage allocation and the fragmentation of computer memory. In the context of queueing theory it is referred to as an $\mathrm{M} / \mathrm{M} / \infty$ queue with ranked servers. It has been studied by many authors including Kosten [8]; Coffman et al. [5], and Newell [9]. In particular, Coffman et al. solved for the steady-state probability distribution of the highest ranked occupied server $(\max S$, if we define $S$ to be the set of occupied servers) using generating functions. However, the solution is given in the form of an alternating sum and it is not easy to understand the solution's qualitative behavior.

Here we are interested in the probability distribution of the wasted spaces $W$, which are defined as the difference between the number of largest occupied spaces $(\max S$ ) and the total number of occupied spaces $(|S|)$. Coffman and Leighton [2] gave an approximation for the mean wasted space using a simple probabilistic proof, and Aldous [1] used more refined probabilistic arguments to obtain a better approximation. In [1] the mean wasted space was shown to be

\footnotetext{
Received 17 December 2007.

* Postal address: Department of Mathematics, Statistics, and Computer Science, University of Illinois at Chicago, 851 South Morgan (M/C 249), Chicago, IL 60607-7045, USA.

** Email address: esohn3@math.uic.edu

*** Email address: kness1@uic.edu

**** This work was partly supported by NSF grant DMS 05-03745.
} 
$\mathrm{E}[W] \sim \sqrt{2 \rho \log \log \rho}, \rho \rightarrow \infty$, and the distribution of max $S$ follows a double exponential (or extreme value) distribution in this limit. More recently, Knessl obtained detailed asymptotic results for $\rho \rightarrow \infty$, first [6] for the distribution of $\max S$, and later [7] for the joint distribution of the numbers of occupied primary and secondary servers.

In [11] we showed that the solution for the joint distribution can be obtained using more elementary methods, by solving the basic difference equation using separation of variables. In this paper we obtain the probability distribution of the wasted spaces using the results in [7] and evaluating asymptotically certain sums and integrals.

Related work includes Preater [10], who gave a new probabilistic derivation for the distribution of $\max S$, by reducing the problem to the solution of a random difference equation. Similar models with various service disciplines and storage policies have been studied in [3] and [4]. In [4] precise estimates were given for the mean wasted spaces in ranked server $M / M / 1$ models under the first-in-first-out (FIFO) and processor sharing (PS) service disciplines. In [3] some alternate storage policies were considered, where an arrival does not necessarily take the lowest ranked server. These lead to more tractable mathematical models, which provide upper and lower bounds for the more difficult FIFO and PS M/M/1 models.

The paper is organized as follows. In Section 2 we summarize the main results. The derivations are sketched in Section 3. In Section 4 we include numerical studies and comparisons.

\section{Summary of results}

We let $N_{1}(t)$ and $N_{2}(t)$ respectively denote the number of primary and secondary servers occupied at time $t$. The joint steady-state distribution function is

$$
\pi(k, r)=\pi(k, r ; m)=\lim _{t \rightarrow \infty} \operatorname{Pr}\left[N_{1}(t)=k, N_{2}(t)=r\right], \quad 0 \leq k \leq m, r \geq 0 .
$$

Let $\rho=\lambda_{0} / \mu$ be the traffic intensity. In [7] and [11] we obtained the integral representation

$$
\pi(k, r)=\frac{m !}{r ! k !} \rho^{k-m} \frac{1}{2 \pi \mathrm{i}} \int_{B r} \rho^{-z} \Gamma(z+r) \frac{G_{k}(z)}{G_{m}(z) G_{m}(z-1)} \mathrm{d} z,
$$

where $G_{k}$ is the polynomial

$$
\begin{aligned}
G_{k}(z) & =G_{k}(z ; \rho) \\
& =\sum_{L=0}^{k}\left(\begin{array}{l}
k \\
L
\end{array}\right) \rho^{-L} \frac{\Gamma(L-z)}{\Gamma(-z)} \\
& =\frac{k !}{\rho^{k}} \frac{1}{2 \pi \mathrm{i}} \int_{C} t^{-k-1} \mathrm{e}^{\rho t}(1-t)^{z} \mathrm{~d} t .
\end{aligned}
$$

Here $\Gamma(\cdot)$ is the gamma function, the contour $C$ is a small loop about the origin in the complex $t$-plane, and the contour $\mathrm{Br}$ is the vertical Bromwich contour in the $z$-plane. We can take $\mathrm{Br}$ to be the imaginary axis for $r \geq 1$ and, for $r=0$, we replace $B r$ by $B r_{+}$, which goes along the imaginary axis with an indentation to the right of $z=0$.

The probability distribution of wasted space $W$ can be written in terms of $\pi$ as

$$
\operatorname{Pr}[W=0]=\sum_{j=0}^{\infty} \pi(j, 0 ; j)
$$




$$
\operatorname{Pr}[W=L]=\sum_{j=0}^{\infty}(\pi(j, 0 ; L+j)-\pi(j, 0 ; L+j-1)), \quad L \geq 1 .
$$

We also have the alternate forms

$$
\operatorname{Pr}[W \leq L]=\sum_{j=0}^{\infty} \pi(j, 0 ; L+j)
$$

and

$$
\operatorname{Pr}[W>L]=\sum_{j=0}^{\infty}\left(\frac{\rho^{j}}{j !} \mathrm{e}^{-\rho}-\pi(j, 0 ; L+j)\right) .
$$

The last expression will be useful in estimating the right tail of the distribution. In some cases $\pi$ will be very close to a Poisson distribution and it will prove essential to estimate carefully the summand in (2.6), which measures the deviation from this distribution.

Using the integral representation in (2.1) and the asymptotic results in [7], we obtain asymptotic results for the probability distribution of wasted spaces, as summarized below.

Theorem 2.1. For $\rho \rightarrow \infty$ and $\lambda=\sqrt{2 \log \log \rho}$, the distribution of wasted spaces, $\operatorname{Pr}[W=$ $L]$, has the following asymptotic behaviors.

(i) For $L=0$,

$$
\operatorname{Pr}[W=0] \sim \frac{\sqrt{2}}{\sqrt{\rho} \log \rho \sqrt{\log \log \rho}} .
$$

(ii) For $L=\sqrt{\rho} \lambda(1-U)$ and $-1<U \leq 1$,

$$
\operatorname{Pr}[W=L] \sim \frac{1}{\sqrt{2 \pi \rho}} \frac{\Gamma(U+1)}{(\lambda A)^{U}} \exp \left[-\frac{1}{2} \lambda^{2} U^{2}\right],
$$

where $A=1 /(2 \sqrt{2 \pi})$.

(iii) For $L=\sqrt{\rho}\left(2 \lambda+\Lambda_{*}\right)$ and $\Lambda_{*}=O(1)$,

$$
\operatorname{Pr}[W=L] \sim \frac{\log \log \rho}{2 \pi \sqrt{2 \rho} \log \rho} \exp \left[-\lambda \Lambda_{*}-\frac{\Lambda_{*}^{2}}{4}\right] \int_{-\infty}^{\Lambda_{*} / \sqrt{2}} \exp \left[-\frac{\xi^{2}}{2}\right] \mathrm{d} \xi
$$

(iv) For $L=\rho^{2 / 3} \zeta$ and $\zeta=O(1)$,

$$
\operatorname{Pr}[W=L] \sim \frac{\zeta^{2}}{8 \sqrt{\pi} \rho^{1 / 6}}\left(\exp \left[\frac{\zeta^{3}}{8}\right] \int_{\zeta^{3} / 8}^{\infty} \frac{\mathrm{e}^{-u}}{u} \mathrm{~d} u\right) \exp \left[-\frac{1}{4} \rho^{1 / 3} \zeta^{2}\right] .
$$

(v) For $L=v \rho$ and $v=O(1)$,

$$
\begin{gathered}
\operatorname{Pr}[W=L] \sim \frac{1}{4 \sqrt{\pi \rho}} \frac{2+v-\sqrt{4+v^{2}}}{\sqrt{4+v^{2}}-2}\left(1+\frac{v^{2}}{4}\right)^{-1 / 4} \mathrm{e}^{\rho \phi(v)}, \\
\phi(v)=-2+\sqrt{v^{2}+4}-v \log \left(\sqrt{1+\frac{v^{2}}{4}}+\frac{v}{2}\right) .
\end{gathered}
$$


We can simplify (2.8) in two special cases. If $L=\sqrt{\rho}\left(\lambda+\Lambda_{1}\right)$ with $\Lambda_{1}=O(1)$, corresponding to $U=O\left(\lambda^{-1}\right)$, we obtain the Gaussian limit law:

$$
\operatorname{Pr}[W=L] \sim \frac{1}{\sqrt{2 \pi \rho}} \exp \left[-\frac{\Lambda_{1}^{2}}{2}\right], \quad \Lambda_{1}=\frac{L}{\sqrt{\rho}}-\sqrt{2 \log \log \rho} .
$$

If, on the other hand, $U \rightarrow 1$ with $1-U=\Lambda^{\prime} / \lambda^{2}=O\left(\lambda^{-2}\right)$, from (2.8) we obtain

$$
\operatorname{Pr}[W=L] \sim \frac{2}{\sqrt{\rho} \log \rho \sqrt{2 \log \log \rho}} \mathrm{e}^{\Lambda^{\prime}}, \quad \Lambda^{\prime}=\frac{L}{\sqrt{\rho}} \sqrt{2 \log \log \rho} .
$$

On this scale, the distribution grows exponentially. Note that (2.7) is a special case of (2.8) (with $U=1$ ) and (2.12) (with $\Lambda^{\prime}=0$ ), but we wanted to explicitly display the probability of having zero wasted space.

In (2.7)-(2.9) the probability is, up to some logarithmic factors, roughly $O\left(\rho^{-1 / 2}\right)$. Note that $\exp \left[-\lambda^{2} U^{2} / 2\right]=(\log \rho)^{-U^{2}}$. We can view $0<U<1$ in $(2.8)$ as the left tail of the distribution and $-1<U<0$ as the 'near-right' tail. In (2.10) and (2.11) the distribution is exponentially small in $\rho^{1 / 3}$ and $\rho$, respectively, and we call these the right tail and the farright tail. It can be easily shown that (2.8) and (2.9) match in the intermediate limit where $U \downarrow-1$ and $\Lambda_{*}=-\lambda-\lambda U \rightarrow-\infty$, that (2.9) and (2.10) match as $\Lambda_{*} \rightarrow+\infty$ and $\zeta \rightarrow 0$ $\left(\zeta=\rho^{-1 / 6}\left(2 \lambda+\Lambda_{*}\right)\right)$, and that (2.10) matches to (2.11) as $\zeta \rightarrow \infty$ and $v \rightarrow 0\left(v=\rho^{-1 / 3} \zeta\right)$.

We will also show that, for a fixed $\rho$ and $L \rightarrow \infty$, we have

$$
\operatorname{Pr}[W=L] \sim \operatorname{Pr}[W \geq L] \sim \frac{\rho^{L+1}}{L(L !)} \mathrm{e}^{-2 \rho} .
$$

This gives the tail behavior for moderate traffic intensities. In Section 3 we will also establish the asymptotic matching of (2.13) as $\rho \rightarrow \infty$ with (2.11) as $v=L / \rho \rightarrow \infty$.

\section{Derivations}

Knessl [6], [7] gave detailed asymptotic results for $G_{k}(z)$ and $\pi(k, r ; m)$ for $\rho \rightarrow \infty$ and all possible ranges of $k, r$, and $m$. Here we use these results, setting $r=0, k=j$, and $m=L+j$, to asymptotically evaluate the sums in (2.3)-(2.6). We will ultimately get different expansions for four different ranges of $L$, which will lead to Theorem 2.1. When using (2.3)-(2.6), we need to determine where the summands (e.g. $\pi(j, 0 ; j)$ in (2.3)) are maximal as functions of $j$ for $\rho \rightarrow \infty$. This will also depend on the size of $L$ with respect to $\rho$. We first consider the case in which $L=0$.

When $L=0,(2.1)$ and (2.3) combine to give the integral representation.

$$
\begin{aligned}
\operatorname{Pr}[W=0] & =\sum_{j=0}^{\infty} \frac{1}{2 \pi \mathrm{i}} \int_{B r_{+}} \frac{\Gamma(z) \rho^{-z}}{G_{j}(z-1)} \mathrm{d} z \\
& =\sum_{j=0}^{\infty} \frac{1}{2 \pi \mathrm{i}} \int_{B r_{+}^{\prime}} \frac{z \Gamma(z) \rho^{-z-1}}{G_{j}(z)} \mathrm{d} z .
\end{aligned}
$$

The contour $\mathrm{Br}_{+}^{\prime}$ is obtained by shifting $B r_{+}$to the left by one unit in the complex $z$-plane. 
Using the asymptotic results for $G_{k}$ in [6] and evaluating the integral(s) in (3.1) for $\rho \rightarrow \infty$ and various ranges of $j$, we find that the summand is concentrated where $j=\rho+O(\sqrt{\rho})$. Then approximating the sum over $j$ by an integral over $\alpha=(j-\rho) / \sqrt{\rho}$ we obtain

$$
\operatorname{Pr}[W=0] \sim \int_{-\infty}^{\infty} \exp \left[-\frac{\alpha^{2}}{4}\right] \rho^{-z_{0} / 2} \frac{z_{0} \Gamma\left(z_{0}\right)}{\sqrt{\rho} \Delta(\alpha)} \mathrm{d} \alpha .
$$

Here $\Delta(\alpha)=-\left.(\mathrm{d} / \mathrm{d} z) D_{z}(-\alpha)\right|_{z=z_{0}(\alpha)}$, where $D_{z}$ is the parabolic cylinder function of index $z$ and $z=z_{0}(\alpha)$ is the smallest positive root of $D_{z}(-\alpha)$.

The integral in (3.2) still contains the large parameter $\rho$ and the integrand varies algebraically with $\rho$ (we note that, away from the range $j=\rho+O(\sqrt{\rho})$, the summand in (3.1) is exponentially small). We can therefore further simplify (3.2). Since $z_{0}>0$, the factor $\rho^{-z_{0} / 2}$ is largest where $z_{0}$ is smallest, and this occurs for $\alpha \rightarrow \infty$. In this limit the maximal root of $D_{z}(-\alpha)$ satisfies

$$
z_{0}(\alpha) \sim \frac{\alpha}{\sqrt{2 \pi}} \exp \left[-\frac{\alpha^{2}}{2}\right], \quad \alpha \rightarrow \infty .
$$

For $\alpha \rightarrow \infty$, we have the asymptotic expansion

$$
D_{z}(-\alpha) \sim(-\alpha)^{z} \exp \left[-\frac{\alpha^{2}}{4}\right]+\frac{\sqrt{2 \pi}}{\Gamma(-z)} \exp \left[\frac{\alpha^{2}}{4}\right] \alpha^{-z-1}, \quad \alpha \rightarrow \infty
$$

The expansion for $z_{0}(\alpha)$ follows from the above, as $\Gamma(-z) \sim-1 / z$ for $z \rightarrow 0$. Then it follows that $\Delta(\alpha) \sim(\sqrt{2 \pi} / \alpha) \exp \left[\alpha^{2} / 4\right]$ and (3.2) becomes

$$
\begin{aligned}
\operatorname{Pr}[W=0] & \sim \int_{-\infty}^{\infty} \frac{\alpha}{\sqrt{2 \pi \rho}} \exp \left[-\frac{\alpha^{2}}{2}\right] \exp \left[-\frac{z_{0} \theta}{2}\right] \mathrm{d} \alpha \\
& \sim \int_{-\infty}^{\infty} \frac{\alpha}{\sqrt{2 \pi \rho}} \exp \left[-\frac{\alpha^{2}}{2}\right] \exp \left[-A \alpha \theta \exp \left[-\frac{\alpha^{2}}{2}\right]\right] \mathrm{d} \alpha, \quad \theta=\log \rho
\end{aligned}
$$

where $A=1 /(2 \sqrt{2 \pi})$. Setting

$$
\alpha=\sqrt{2 \log \theta}+\frac{\log (\sqrt{2 \log \theta})+\omega}{\sqrt{2 \log \theta}},
$$

and using $\mathrm{d} \alpha=\mathrm{d} \omega / \sqrt{2 \log \theta},(3.3)$ becomes

$$
\operatorname{Pr}[W=0] \sim \frac{2}{\theta \sqrt{2 \rho \log \theta}} \int_{-\infty}^{\infty} A \mathrm{e}^{-\omega} \exp \left[-A \mathrm{e}^{-\omega}\right] \mathrm{d} \omega .
$$

The integral is equal to 1 and we thus obtain (2.7), since $\theta=\log \rho$. It is interesting to note that the integrand in (3.4) is a double exponential, which occurs as a limiting distribution of $\max S$ (see [1] and [6]).

For $L \geq 1$, we rewrite (2.4) as

$$
\operatorname{Pr}[W=L]=\sum_{j=0}^{\infty}(\pi(j, 0 ; L+j)-\pi(j+1,0 ; L+j))-\pi(0,0 ; L-1) .
$$


It proves easier to estimate the summand in (3.5), rather than the summand in (2.4). From (2.1) we obtain the following integral representation:

$$
\begin{aligned}
& \pi(j, 0 ; L+j)-\pi(j+1,0 ; L+j) \\
& \quad=\frac{(L+j) !}{j !} \frac{\rho^{-L}}{2 \pi \mathrm{i}} \int_{B r_{+}} \frac{\rho^{-z} \Gamma(z)\left(G_{j}(z)-(\rho /(j+1)) G_{j+1}(z)\right)}{G_{L+j}(z) G_{L+j}(z-1)} \mathrm{d} z .
\end{aligned}
$$

Now we scale $L=\Lambda \sqrt{\rho}=O(\sqrt{\rho})$. By using results in [7] we conclude that the right-hand side of (3.6) is again concentrated in the range $j=\rho+O(\sqrt{\rho})$ and that the integral can be approximated by parabolic cylinder functions. But, when $L=O(\sqrt{\rho}), \Lambda=L / \sqrt{\rho}$ appears explicitly in the arguments of these functions and, thus, affects the location of the minimal root. From [7] we obtain, again setting $\alpha=(j-\rho) / \sqrt{\rho}$,

$$
\begin{aligned}
\pi(j, 0 ; L+j)-\pi(j+1,0 ; L+j) \\
\sim \frac{(L+j) !}{j !} \frac{1}{\rho^{L} \sqrt{\rho}} \exp \left[-\frac{\Lambda^{2}}{2}-\Lambda \alpha-\frac{\alpha^{2}}{4}\right] \\
\quad \times \frac{\Gamma\left(z_{0}\right) \rho^{-z_{0} / 2}\left(\left(z_{0} / \sqrt{\rho}\right) D_{z_{0}-1}(-\alpha)+(1-\rho /(j+1)) D_{z_{0}}(-\alpha)\right)}{D_{z_{0}-1}(-\alpha-\Lambda)\left(-\left.(\mathrm{d} / \mathrm{d} z) D_{z}(-\alpha-\Lambda)\right|_{z=z_{0}(\alpha, \Lambda)}\right)} .
\end{aligned}
$$

Now $z_{0}=z_{0}(\alpha, \Lambda)$ is the smallest positive root of the parabolic cylinder function $D_{z}(-\alpha-\Lambda)$.

Using Stirling's formula in the form

$$
\frac{(L+j) !}{j !} \sim \rho^{L} \mathrm{e}^{\Lambda \alpha} \exp \left[\frac{\Lambda^{2}}{2}\right], \quad j=\rho+\alpha \sqrt{\rho}, L=\Lambda \sqrt{\rho}
$$

and also noting that $1-\rho /(j+1) \sim \alpha / \sqrt{\rho}$, we obtain

$$
\operatorname{Pr}[W=L] \sim \sum_{j=0}^{\infty} \frac{\exp \left[-\alpha^{2} / 4\right]}{\rho} \frac{\Gamma\left(z_{0}\right) \rho^{-z_{0} / 2}\left(z_{0} D_{z_{0}-1}(-\alpha)+\alpha D_{z_{0}}(-\alpha)\right)}{D_{z_{0}-1}(-\alpha-\Lambda)\left(-\left.(\mathrm{d} / \mathrm{d} z) D_{z}(-\alpha-\Lambda)\right|_{z=z_{0}(\alpha, \Lambda)}\right)}
$$

Here we ignored $\pi(0,0 ; L-1)$ in (3.5), since it is exponentially small (in fact, it is equal to $\left.\mathrm{e}^{-\rho}\right)$. Again, approximating the sum by an integral over $\alpha$ and using the identity

$$
z_{0} D_{z_{0}-1}(-\alpha)+\alpha D_{z_{0}}(-\alpha)=-D_{z_{0}+1}(-\alpha)
$$

we are led to

$$
\operatorname{Pr}[W=L] \sim \int_{-\infty}^{\infty} \frac{\exp \left[-\alpha^{2} / 4\right]}{\sqrt{\rho}} \frac{\Gamma\left(z_{0}\right) \rho^{-z_{0} / 2}\left(-D_{z_{0}+1}(-\alpha)\right)}{D_{z_{0}-1}(-\alpha-\Lambda)\left(-\left.(\mathrm{d} / \mathrm{d} z) D_{z}(-\alpha-\Lambda)\right|_{z=z_{0}(\alpha, \Lambda)}\right)} \mathrm{d} \alpha
$$

Note that, when $\Lambda=0$, (3.7) reduces to (3.2). Again, in view of the factor $\rho^{-z_{0} / 2}$, we can simplify the integrand further and obtain a more explicit result. 
Setting $\beta=\alpha+\Lambda$ we now have $z_{0}(\beta) \sim(\beta / \sqrt{2 \pi}) \exp \left[-\beta^{2} / 2\right]$ and

$$
\begin{aligned}
D_{z_{0}-1}(-\beta) & \sim \frac{\sqrt{2 \pi}}{\Gamma\left(-z_{0}+1\right)} \exp \left[\frac{\beta^{2}}{4}\right] \beta^{-z_{0}}, \\
D_{z_{0}+1}(-\beta+\Lambda) \sim & (\Lambda-\beta)^{z_{0}+1} \exp \left[-\frac{(\beta-\Lambda)^{2}}{4}\right] \\
& +\frac{\sqrt{2 \pi}}{\Gamma\left(-z_{0}-1\right)} \exp \left[\frac{(\beta-\Lambda)^{2}}{4}\right](\beta-\Lambda)^{-z_{0}-2}, \\
-\left.\frac{\mathrm{d}}{\mathrm{d} z} D_{z}(-\beta)\right|_{z=z_{0}(\beta)} & \frac{\sqrt{2 \pi}}{\beta} \exp \left[\frac{\beta^{2}}{4}\right] .
\end{aligned}
$$

For $z_{0} \rightarrow 0$, we furthermore use $\Gamma\left(-z_{0}-1\right)=\Gamma\left(-z_{0}\right) /\left(-z_{0}-1\right) \sim-\Gamma\left(-z_{0}\right) \sim 1 / z_{0}$ and $\Gamma\left(z_{0}\right)=\Gamma\left(z_{0}+1\right) / z_{0} \sim 1 / z_{0}$, so that the first term on the right-hand side of (3.9) is asymptotically larger than the second term. Thus, using (3.8)-(3.10), (3.7) simplifies to

$$
\operatorname{Pr}[W=L] \sim \frac{1}{\sqrt{2 \pi \rho}} \int_{-\infty}^{\infty} \rho^{-z_{0} / 2}(\beta-\Lambda) \exp \left[-\frac{(\beta-\Lambda)^{2}}{2}\right] \mathrm{d} \beta .
$$

Note that this approximation assumes that $\Lambda=O(1)$ and that $\beta$ is large. We now evaluate (3.11) in various limits and show that the major contribution indeed comes from large values of $\beta$.

We replace $z_{0}$ in (3.11) by its asymptotic expansion $z_{0} \sim(\beta / \sqrt{2 \pi}) \exp \left[-\beta^{2} / 2\right]$, and set

$$
\beta=\lambda+\varrho, \quad \Lambda=\lambda(1-U), \quad \text { and } \quad \lambda=\sqrt{2 \log \log \rho}=\sqrt{2 \log \theta} .
$$

Then, in terms of $\varrho$ and $U,(3.11)$ becomes

$$
\operatorname{Pr}[W=L] \sim \frac{1}{\sqrt{2 \pi \rho}} \int_{-\infty}^{\infty}(\varrho+\lambda U) \exp \left[-\frac{(\varrho+\lambda U)^{2}}{2}\right] \exp \left[-A \lambda \mathrm{e}^{-\lambda \varrho}\right] \mathrm{d} \varrho .
$$

For $\lambda \rightarrow \infty$, the maximum of the integrand occurs when $-\lambda \varrho=\log \left((\lambda U+\varrho) /\left(A \lambda^{2}\right)\right)$. If we scale $\varrho$ as $\varrho=-(1 / \lambda) \log (U /(A \lambda))+v / \lambda$, (3.12) becomes

$$
\operatorname{Pr}[W=L] \sim \frac{U}{\sqrt{2 \pi \rho}} \exp \left[-\frac{\lambda^{2} U^{2}}{2}\right]\left(\frac{U}{A \lambda}\right)^{U} \int_{-\infty}^{\infty} \exp \left[-U\left(v+\mathrm{e}^{-v}\right)\right] \mathrm{d} v .
$$

The integral evaluates to $U^{-U} \Gamma(U)$. We thus obtain (2.8) for $U>0$ if we write $U \Gamma(U)=$ $\Gamma(U+1)$. We can show that this expression remains valid for $-1<U \leq 0$, using an initial integration by parts in (3.11). However, the approximation in (2.8) leads to a singularity as $U \downarrow-1$, which corresponds to $L$ increasing past $2 \lambda \sqrt{\rho}=2 \sqrt{\rho} \sqrt{2 \log \log \rho}$. Thus, there is a transition in the asymptotic behavior when the wasted space is approximately twice its (asymptotic) mean value.

To study this transition, we return to (3.11) and replace $\rho^{-z_{0} / 2}$ in (3.11) by

$$
\exp \left[-A \theta \beta \exp \left[-\frac{\beta^{2}}{2}\right]\right]
$$

and then integrate by parts using

$$
\mathrm{d}\left(\exp \left[-\frac{(\beta-\Lambda)^{2}}{2}\right]\right)=-(\beta-\Lambda) \exp \left[-\frac{(\beta-\Lambda)^{2}}{2}\right]
$$


Then we let $\Lambda=2 \lambda+\Lambda_{*}$ with $\Lambda_{*}=O(1)$, and let $\beta=\lambda+x$ with $x=O(1)$. Thus, we obtain

$$
\begin{gathered}
\frac{A \theta}{\sqrt{2 \pi \rho}} \int_{-\infty}^{\infty}\left((\lambda+x)^{2}-1\right) \exp \left[-\left(x-\frac{\Lambda_{*}}{2}\right)^{2}-\frac{1}{4}\left(2 \lambda+\Lambda_{*}\right)^{2}\right] \\
\times \exp \left[-A(\lambda+x) \exp \left[-\lambda x-\frac{x^{2}}{2}\right]\right] \mathrm{d} x .
\end{gathered}
$$

As $\lambda \rightarrow \infty$, the last exponential factor behaves as a step function, since

$$
\exp \left[-A(\lambda+x) \exp \left[-\lambda x-\frac{x^{2}}{2}\right]\right] \rightarrow \begin{cases}1, & x>0 \\ 0, & x<0\end{cases}
$$

Using $\theta \exp \left[-\lambda^{2}\right]=1 / \log \rho$, the above integral asymptotically simplifies to

$$
\frac{A \lambda^{2}}{\sqrt{2 \pi \rho} \log \rho} \exp \left[-\lambda \Lambda_{*}-\frac{\Lambda_{*}^{2}}{4}\right] \int_{0}^{\infty} \exp \left[-\left(x-\frac{\Lambda_{*}}{2}\right)^{2}\right] \mathrm{d} x .
$$

From the above we obtain (2.9) if we change the integration variable $x$ to $\frac{1}{2}\left(\Lambda_{*}-\sqrt{2} \xi\right)$.

Next we consider the case in which $L=O(\rho)$. The previous results applied where (roughly) $L=O(\sqrt{\rho})$, and, on these scales, $\operatorname{Pr}[W=L]$ is (roughly) $O(1 / \sqrt{\rho})$. But now we are going into the right tail of the distribution, where we can expect the probabilities to be very small. It proves useful to use form (2.6) for $\operatorname{Pr}[W>L]$. Knessl [7] estimated $\pi(k, r ; m)$ when $r=0$, $k=\rho X$, and $m=\rho X_{0}$. For $X_{0}>1$ and $X<1$, Knessl obtained

$$
\begin{gathered}
\frac{\rho^{j} \mathrm{e}^{-\rho}}{j !}-\pi(j, 0 ; L+j) \sim \frac{\sqrt{X}}{2 \pi \rho \sqrt{X_{0}}} \frac{1}{(1-X)\left(X_{0}-1\right)} \exp \left[\rho \Psi\left(X, X_{0}\right)\right], \\
\Psi\left(X, X_{0}\right)=-2+X+X_{0}-X \log X-X_{0} \log X_{0} .
\end{gathered}
$$

Here we replace $j$ by $\rho X$ and $L+j$ by $\rho X_{0}$. Using (3.14) in (2.6) leads to

$$
\operatorname{Pr}[W>L] \sim \sum_{j=0}^{\infty} \frac{1}{2 \pi \rho} \sqrt{\frac{j}{L+j}} \frac{1}{(1-j / \rho)((L+j) / \rho-1)} \exp \left[\rho \Psi\left(\frac{j}{\rho}, \frac{L+j}{\rho}\right)\right] .
$$

The sum in (3.15) can be estimated by the discrete Laplace method, with the main contribution coming from where $\Psi(j / \rho,(L+j) / \rho)$ is maximal in $j$, which occurs when $j(L+j)=\rho^{2}$ or

$$
j=\rho \zeta_{*}, \quad \text { where } \quad \zeta_{*}=-\frac{v}{2}+\sqrt{1+\frac{v^{2}}{4}} \quad \text { and } \quad v=\frac{L}{\rho} .
$$

We take $v$ to be $O(1)$. We can estimate (3.15) by approximating the summand for $j=$ $\rho \zeta_{*}+O(\sqrt{\rho})$ and approximating the sum by an integral. But, a quicker derivation is obtained by using Stirling's formula in reverse, in the form(s)

$$
\begin{gathered}
\mathrm{e}^{j-\rho}\left(\frac{\rho}{j}\right)^{j} \sim \sqrt{2 \pi j} \frac{\rho^{j} \mathrm{e}^{-\rho}}{j !}, \\
\mathrm{e}^{-\rho} \mathrm{e}^{L+j}\left(\frac{\rho}{L+j}\right)^{L+j} \sim \sqrt{2 \pi(L+j)} \frac{\rho^{L+j} \mathrm{e}^{-\rho}}{(L+j) !} .
\end{gathered}
$$


We use the above in (3.15) to obtain

$$
\operatorname{Pr}[W>L] \sim \frac{\zeta_{*}}{\left(1-\zeta_{*}\right)\left(v+\zeta_{*}-1\right)} \sum_{j=0}^{\infty} \frac{\rho^{L+2 j} \mathrm{e}^{-2 \rho}}{j !(L+j) !} .
$$

Here we also froze the factor $(1-j / \rho)((L+j) / \rho-1)$ at the maximum. The sum in (3.16) can be recognized as a modified Bessel function, since

$$
\sum_{l=0}^{\infty} \frac{Y^{2 l}}{l !(L+l) !}=Y^{-L} I_{L}(2 Y)
$$

We thus obtain

$$
\operatorname{Pr}[W>L] \sim \frac{\zeta_{*}}{\left(1-\zeta_{*}\right)\left(v+\zeta_{*}-1\right)} \mathrm{e}^{-2 \rho} I_{L}(2 \rho)
$$

For $L$ and $\rho$ simultaneously large, with $L / \rho=v=O(1)$, we have

$$
\begin{gathered}
I_{L}(2 \rho) \sim \frac{1}{2 \sqrt{\pi \rho}}\left(1+\frac{v^{2}}{4}\right)^{-1 / 4} \mathrm{e}^{\rho \psi(v)}, \\
\psi(\nu)=\sqrt{v^{2}+4}-v \log \left(\sqrt{1+\frac{v^{2}}{4}}+\frac{v}{2}\right) .
\end{gathered}
$$

Using (3.18) in (3.17), defining $C \equiv \sqrt{1+4 / \nu^{2}}$, and noting that $\zeta_{*}=v(C-1) / 2$, we obtain

$$
\operatorname{Pr}[W>L] \sim \frac{\sqrt{2}}{4 \sqrt{\pi \rho}} \frac{(C-1) \sqrt{\nu}}{(\nu C-2) \sqrt{C}} \exp \left[\rho\left(\nu C-v \log \left(\frac{v}{2}(C+1)\right)-2\right)\right],
$$

where $C=\sqrt{1+4 / v^{2}}$. We define $\phi(v)$ and $f(v)$ by

$$
\begin{aligned}
\phi(v) & =v C-v \log \left(\frac{v(C+1)}{2}\right)-2 \\
& =-2+\sqrt{v^{2}+4}-v \log \left(\sqrt{\frac{v^{2}}{4}+1}+\frac{v}{2}\right), \\
f(v) & =\frac{\sqrt{2}}{4 \sqrt{\pi \rho}} \frac{(C-1) \sqrt{v}}{(v C-2) \sqrt{C}},
\end{aligned}
$$

and note that $\phi(v)=\psi(v)-2$. Then we can write

$$
\begin{aligned}
\operatorname{Pr}[W=L] & =\operatorname{Pr}[W>L-1]-\operatorname{Pr}[W>L] \\
& \sim f\left(v-\frac{1}{\rho}\right) \mathrm{e}^{\rho \phi(v-1 / \rho)}-f(v) \mathrm{e}^{\rho \phi(v)} \\
& \sim f(v) \mathrm{e}^{\rho \phi(v)}\left(\mathrm{e}^{-\phi^{\prime}(v)}-1\right) \\
& \sim \operatorname{Pr}[W>L]\left(\mathrm{e}^{-\phi^{\prime}(v)}-1\right) .
\end{aligned}
$$

Since $\phi^{\prime}(v)=-\log \left(\sqrt{1+v^{2} / 4}+v / 2\right)$, we obtain $(2.11)$. 
Next we show that (2.9) cannot asymptotically match to (2.11). If we let $v \rightarrow 0$ in (2.11) and use $\phi(v) \sim-v^{2} / 4$, we obtain

$$
\operatorname{Pr}[W=L] \sim \frac{\exp \left[-\rho v^{2} / 4\right]}{v \sqrt{\pi \rho}}=\frac{\sqrt{\rho}}{L \sqrt{\pi}} \exp \left[-\frac{L^{2}}{4 \rho}\right] .
$$

However, if we let $\Lambda_{*} \rightarrow \infty$ in (2.9) and then set $\Lambda_{*}=L / \sqrt{\rho}-2 \lambda$, we obtain

$$
\operatorname{Pr}[W=L] \sim \frac{\log \rho \log \log \rho}{2 \sqrt{\pi \rho}} \exp \left[-\frac{L^{2}}{4 \rho}\right] .
$$

While the exponential factors in (3.20) and (3.21) agree, the algebraic factors do not. This indicates that another scale is needed, which will correspond to $L-2 \lambda \sqrt{\rho} \gg O(\sqrt{\rho})$ and $L \ll O(\rho)$.

To identify the new scale and obtain the corresponding expansion of $\operatorname{Pr}[W=L]$, we first re-examine some of the results in [7]. There Knessl showed that, for $r=0, m=\rho+O(\sqrt{\rho})$, and $k=\rho+O(\sqrt{\rho})$

$$
\pi(k, 0 ; m) \sim \frac{\Gamma\left(z_{0}\right)}{\Delta(\beta)} \frac{D_{z_{0}}(-\alpha)}{D_{z_{0}-1}(-\beta)} \frac{\exp \left[-\alpha^{2} / 4\right]}{\sqrt{\rho}} \rho^{-z_{0} / 2},
$$

where

$$
\alpha=\frac{k-\rho}{\sqrt{\rho}}, \quad \beta=\frac{m-\rho}{\sqrt{\rho}},
$$

and $z_{0}(\beta)$ is the minimum positive root of $D_{z}(-\beta)$, as before. In [7] Knessl also showed that, for $X_{0}=m / \rho>1$,

$$
\begin{aligned}
\frac{\rho^{k} \mathrm{e}^{-\rho}}{k !}-\pi(k, 0 ; m) \sim & \frac{1}{2 \pi\left(X_{0}-1\right) \sqrt{\rho X_{0}}}\left(\int_{-\alpha}^{\infty} \exp \left[-\frac{u^{2}}{2}\right] \mathrm{d} u\right) \\
& \times \exp \left[\rho\left(-1+X_{0}-X_{0} \log X_{0}\right)\right] .
\end{aligned}
$$

If we expand (3.22) for $\beta \rightarrow \infty$, noting that $z_{0} \rightarrow 0$ with

$$
\begin{gathered}
z_{0} \sim \frac{\beta}{\sqrt{2 \pi}} \exp \left[-\frac{\beta^{2}}{2}\right], \quad \Delta(\beta) \sim \frac{\sqrt{2 \pi}}{\beta} \exp \left[\frac{\beta^{2}}{4}\right], \\
D_{z_{0}}(-\alpha) \sim D_{0}(-\alpha)=\exp \left[-\frac{\alpha^{2}}{4}\right],
\end{gathered}
$$

and $\rho^{-z_{0} / 2} \sim 1-\frac{1}{2} z_{0} \log \rho$, the right-hand side of (3.22) becomes

$$
\frac{1}{\sqrt{2 \pi \rho}} \exp \left[-\frac{\alpha^{2}}{2}\right]\left(1-\frac{\beta \log \rho}{2 \sqrt{2 \pi}} \exp \left[-\frac{\beta^{2}}{2}\right]\right), \quad \beta \rightarrow \infty, \frac{m}{\rho} \rightarrow 1 .
$$

Expanding the right-hand side of (3.23) for $X_{0} \rightarrow 1$ and noting that $X_{0}-1=\beta / \sqrt{\rho}$ yields

$$
\frac{1}{2 \pi \beta}\left(\int_{-\alpha}^{\infty} \exp \left[-\frac{u^{2}}{2}\right] \mathrm{d} u\right) \exp \left[-\frac{\beta^{2}}{2}\right], \quad \beta \rightarrow \infty, \frac{m}{\rho} \rightarrow 1 .
$$


Now

$$
\frac{\rho^{k} \mathrm{e}^{-\rho}}{k !} \sim \frac{\exp \left[-\alpha^{2} / 2\right]}{\sqrt{2 \pi \rho}},
$$

so if (3.22) and (3.23) were to asymptotically match, (3.24) would need to agree with

$$
\frac{\beta \log \rho}{4 \pi \sqrt{\rho}} \exp \left[-\frac{\alpha^{2}}{2}\right] \exp \left[-\frac{\beta^{2}}{2}\right], \quad \beta \rightarrow \infty, \frac{m}{\rho} \rightarrow 1,
$$

which is clearly not the case. However, comparing (3.24) and (3.25), we see that the expressions are roughly of comparable magnitude when $\beta / \sqrt{\rho}=O\left(\beta^{-1}\right)$ or $\beta=O\left(\rho^{1 / 4}\right)$. This suggests the scale for the intermediate expansion that is needed between (3.22) (where $\beta=O(1)$ ) and (3.23) (where $\beta=O(\sqrt{\rho})$ ).

We start with the approximation

$$
\pi(k, 0 ; m) \sim \frac{1}{2 \pi \mathrm{i}}\left(\int_{B r_{+}} \frac{\Gamma(z) D_{z}(-\alpha)}{D_{z-1}(-\beta) D_{z}(-\beta)} \rho^{-z / 2} \mathrm{~d} z\right) \frac{\exp \left[-\alpha^{2} / 4\right]}{\sqrt{\rho}},
$$

which can be shown to hold for large $\beta$ as long as $\beta=o(\sqrt{\rho})$. Note that (3.22) is obtained by locating the pole of the integrand in (3.26) in the right-hand half-plane that is closest to the origin, and shifting $B r_{+}$to the right, past this pole. We can also close $B r_{+}$in the left-hand half-plane, and write the integral as a residue series. In the left-hand half-plane there are poles at $z=0,-1,-2, \ldots$, where $\Gamma(z)$ is singular. We thus obtain the alternate form

$$
\begin{aligned}
\pi(k, 0 ; m) \sim & \left(\sum_{l=0}^{\infty} \frac{D_{-l}(-\alpha)}{D_{-l-1}(-\beta) D_{-l}(-\beta)} \frac{\rho^{l / 2}(-1)^{l}}{l !}\right) \frac{\exp \left[-\alpha^{2} / 4\right]}{\sqrt{\rho}} \\
= & \frac{\exp \left[-\alpha^{2} / 2\right]}{\sqrt{\rho}}\left(\int_{-\infty}^{\beta} \exp \left[-\frac{u^{2}}{2}\right] \mathrm{d} u\right)^{-1} \\
& +\left(\sum_{l=1}^{\infty} \frac{D_{-l}(-\alpha)}{D_{-l-1}(-\beta) D_{-l}(-\beta)} \frac{\rho^{l / 2}(-1)^{l}}{l !}\right) \frac{\exp \left[-\alpha^{2} / 4\right]}{\sqrt{\rho}}
\end{aligned}
$$

where in the last expression we isolated the term with $l=0$. Next we use

$$
\begin{gathered}
D_{-l}(-\beta) \sim \beta^{l-1} \exp \left[\frac{\beta^{2}}{4}\right] \frac{\sqrt{2 \pi}}{(l-1) !}, \quad l \geq 1, \beta \rightarrow \infty, \\
\int_{-\infty}^{\beta} \exp \left[-\frac{u^{2}}{2}\right] \mathrm{d} u \sim \sqrt{2 \pi}\left(1-\frac{\exp \left[-\beta^{2} / 2\right]}{\sqrt{2 \pi} \beta}\right), \quad \beta \rightarrow \infty,
\end{gathered}
$$

with which we can rewrite (3.27) in the form

$$
\begin{aligned}
\pi(k, 0 ; m) & -\frac{\exp \left[-\alpha^{2} / 2\right]}{\sqrt{2 \pi \rho}} \\
\sim & \frac{\exp \left[-\beta^{2} / 2\right]}{2 \pi \beta} \frac{\exp \left[-\alpha^{2} / 2\right]}{\sqrt{\rho}} \\
& +\left(\sum_{l=1}^{\infty} \frac{(\sqrt{\rho})^{l}}{\beta^{2 l-1}}(-1)^{l}(l-1) ! D_{-l}(-\alpha)\right) \frac{\exp \left[-\alpha^{2} / 4\right] \exp \left[-\beta^{2} / 2\right]}{2 \pi \sqrt{\rho}} .
\end{aligned}
$$


Now we let

$$
\diamond=\frac{\sqrt{\rho}}{\beta^{2}}=O(1)
$$

and represent $D_{-l}(-\alpha)$ as the integral

$$
D_{-l}(-\alpha)=\frac{\exp \left[\alpha^{2} / 4\right]}{(l-1) !} \int_{-\alpha}^{\infty}(v+\alpha)^{l-1} \exp \left[-\frac{v^{2}}{2}\right] \mathrm{d} v, \quad l \geq 1 .
$$

Using (3.29) in (3.28) and evaluating explicitly the sum over $l$, we obtain

$$
\frac{\exp \left[-\alpha^{2} / 2\right]}{\sqrt{2 \pi \rho}}-\pi(k, 0 ; m) \sim \frac{\exp \left[-\beta^{2} / 2\right]}{2 \pi \beta} \int_{-\alpha}^{\infty} \frac{\exp \left[-v^{2} / 2\right]}{1+\diamond(v+\alpha)} \mathrm{d} v .
$$

Note that the first term on the right-hand side of (3.28) becomes negligible compared to the sum. This is the expression between (3.22) and (3.23) that we sought. As $\diamond \rightarrow 0$ (corresponding to $\left.\beta \gg \rho^{1 / 4}\right)$, the right-hand side of (3.30) becomes the same as (3.24), which shows that (3.23) and (3.30) match. For $\diamond \rightarrow \infty$, we have

$$
\int_{-\alpha}^{\infty} \frac{\exp \left[-v^{2} / 2\right]}{1+\diamond(v+\alpha)} \mathrm{d} v \sim \frac{\log \diamond}{\diamond} \exp \left[-\frac{\alpha^{2}}{2}\right] \sim \frac{\log \rho}{2} \frac{\beta^{2}}{\sqrt{\rho}} \exp \left[-\frac{\alpha^{2}}{2}\right] .
$$

Using (3.30) and (3.31), we see that the result agrees with (3.25), which shows that (3.22) and (3.30) match.

Now we use the new asymptotic result (3.30) to obtain (2.10) in Theorem 2.1. We replace $\alpha$ by $(j-\rho) / \sqrt{\rho}$ and $\beta$ by $(L+j-\rho) / \sqrt{\rho}$, and use $\Lambda=L / \sqrt{\rho}$, to obtain

$$
\begin{aligned}
& \operatorname{Pr}[W>L] \sim \sum_{j=0}^{\infty} \frac{\exp \left[-(L+j-\rho)^{2} /(2 \rho)\right] \sqrt{\rho}}{2 \pi(L+j-\rho)} \\
& \times \int_{0}^{\infty} \frac{1}{1+\diamond v} \exp \left[-\frac{1}{2}\left(v-\frac{j-\rho}{\sqrt{\rho}}\right)^{2}\right] \mathrm{d} v \\
& \sim \int_{-\infty}^{\infty} \frac{\sqrt{\rho}}{2 \pi} \frac{\exp \left[-w^{2} / 2\right]}{w+\Lambda} \exp \left[-\frac{1}{2}(w+\Lambda)^{2}\right] \\
& \times \int_{0}^{\infty} \frac{\mathrm{e}^{w v} \exp \left[-v^{2} / 2\right]}{1+v \sqrt{\rho}(w+\Lambda)^{-2}} \mathrm{~d} v \mathrm{~d} w .
\end{aligned}
$$

Here we set $\diamond=\rho^{3 / 2}(L+j-\rho)^{-2}$ and approximated the sum over $j$ by an integral. We can simplify the double integral in (3.32) as follows, which will also identify the appropriate scaling of $L$. We note that the various exponential factors in the integrand in (3.32) combine to give

$$
\begin{aligned}
& \exp \left[-\frac{1}{2}\left(w+\frac{L}{\sqrt{\rho}}\right)^{2}-\frac{w^{2}}{2}+w v-\frac{v^{2}}{2}\right] \\
& =\mathrm{e}^{v w} \exp \left[-\frac{v^{2}}{2}\right] \exp \left[-\frac{L^{2}}{4 \rho}\right] \exp \left[-\left(w+\frac{L}{2 \sqrt{\rho}}\right)^{2}\right]
\end{aligned}
$$


Scaling $w=-\frac{1}{2} \Lambda+y$ leads to

$$
\begin{aligned}
\operatorname{Pr}[W>L] \sim \frac{\sqrt{\rho}}{2 \pi} \exp \left[-\frac{L^{2}}{4 \rho}\right] \int_{-\infty}^{\infty} \int_{0}^{\infty} & \frac{\exp \left[-y^{2}\right] \exp \left[-v^{2} / 2\right] \mathrm{e}^{v y}}{y+\Lambda / 2} \\
& \times \frac{\mathrm{e}^{-v \Lambda / 2}}{1+v \sqrt{\rho}(y+\Lambda / 2)^{-2}} \mathrm{~d} v \mathrm{~d} y .
\end{aligned}
$$

Furthermore, scaling $v$ to be small, with $v=\varpi \rho^{-1 / 6}$, and $L$ large, with $L=\rho^{2 / 3} \zeta$ (thus, $\left.\Lambda=\rho^{1 / 6} \zeta=O\left(\rho^{1 / 6}\right)\right)$, we see that

$$
v \sqrt{\rho}\left(y+\frac{\Lambda}{2}\right)^{-2} \sim 4 \varpi \zeta^{-2} \text { and } v L \rho^{-1 / 2}=v \Lambda=\varpi \zeta .
$$

Then we can approximate $y+\frac{1}{2} \Lambda$ by $\frac{1}{2} \rho^{1 / 6} \zeta$ and also have $\exp \left[-v^{2} / 2\right] \mathrm{e}^{v y} \sim 1$. Thus, from (3.33), we obtain

$$
\operatorname{Pr}[W>L] \sim \frac{\rho^{1 / 6}}{\pi \zeta}\left(\int_{-\infty}^{\infty} \int_{0}^{\infty} \frac{\exp \left[-y^{2}\right] \mathrm{e}^{-\varpi \zeta / 2}}{1+4 \varpi \zeta^{-2}} \mathrm{~d} \varpi \mathrm{d} y\right) \exp \left[-\frac{1}{4} \rho^{1 / 3} \zeta^{2}\right] .
$$

Upon evaluating the integral over $y$, and rewriting the integral over $\varpi$ by using the substitution $\varpi=-\frac{1}{4} \zeta^{2}+2 \zeta^{-1} u$, from (3.34) we obtain

$$
\operatorname{Pr}[W>L] \sim \frac{\rho^{1 / 6}}{4 \sqrt{\pi}}\left(\zeta \int_{\zeta^{3} / 8}^{\infty} \frac{\mathrm{e}^{-u}}{u} \mathrm{~d} u\right) \exp \left[-\frac{1}{4} \rho^{1 / 3} \zeta^{2}+\frac{1}{8} \zeta^{3}\right] .
$$

To obtain $\operatorname{Pr}[W=L]$, we note that replacing $L$ by $L-1$ corresponds to replacing $\zeta$ by $\zeta-\rho^{-2 / 3}$, and the dominant exponential factor in (3.35) has the asymptotic difference

$$
\begin{aligned}
& \exp \left[-\frac{1}{4} \rho^{1 / 3}\left(\zeta-\rho^{-2 / 3}\right)^{2}\right]-\exp \left[-\frac{1}{4} \rho^{1 / 3} \zeta^{2}\right] \\
& \sim \exp \left[-\frac{1}{4} \rho^{1 / 3} \zeta^{2}\right]\left(\exp \left[\frac{\zeta}{2 \rho^{1 / 3}}\right]-1\right) \\
& \sim \exp \left[-\frac{1}{4} \rho^{1 / 3} \zeta^{2}\right] \frac{\zeta}{2 \rho^{1 / 3}} .
\end{aligned}
$$

We thus obtain (2.10).

As the final step, we verify the asymptotic matching between (2.10) and the other scales. For $\zeta \rightarrow \infty$, we use $\int_{z}^{\infty} u^{-1} \mathrm{e}^{-u} \mathrm{~d} u \sim z^{-1} \mathrm{e}^{-z}$ with $z=\zeta^{3} / 8$ in (3.35), thus obtaining

$$
\operatorname{Pr}[W>L] \sim \frac{2}{\sqrt{\pi}} \rho^{1 / 6} \frac{1}{\zeta^{2}} \exp \left[-\frac{1}{4} \rho^{1 / 3} \zeta^{2}\right]=\frac{2}{\sqrt{\pi \rho}} \frac{1}{v^{2}} \exp \left[-\frac{\rho v^{2}}{4}\right] .
$$

Here we also used $\zeta=\rho^{1 / 3} v$. Now consider (3.19). As $v \rightarrow 0$, we have

$$
C \sim \frac{2}{v}, \quad v C-2 \sim \frac{v^{2}}{4}, \quad \text { and } \quad \phi(v)=-\frac{v^{2}}{4}+O\left(v^{4}\right) .
$$

Thus, (3.19) as $v \rightarrow 0$ becomes the same as (3.36), which verifies the matching between the ranges $L=O\left(\rho^{2 / 3}\right)$ and $L=O(\rho)$. If, on the other hand, $\zeta \rightarrow 0$ then

$$
\int_{\zeta^{3} / 8}^{\infty} \frac{\mathrm{e}^{-u}}{u} \mathrm{~d} u \sim-\log \left(\frac{\zeta^{3}}{8}\right) \sim \frac{1}{2} \log \rho
$$


where we used $\zeta=\left(2 \lambda+\Lambda_{*}\right) \rho^{-1 / 6}$. Thus, for $\zeta \rightarrow 0$, (3.35) becomes

$$
\operatorname{Pr}[W>L] \sim \frac{\lambda \log \rho}{4 \sqrt{\pi}} \exp \left[-\frac{1}{4}\left(\Lambda_{*}+2 \lambda\right)^{2}\right] .
$$

Now $\exp \left[-\lambda^{2}\right]=(\log \rho)^{-2}$ and the expansion of (2.9) as $\Lambda_{*} \rightarrow \infty$ is

$$
\operatorname{Pr}[W=L] \sim \frac{\log \log \rho}{2 \sqrt{\pi \rho} \log \rho} \exp \left[-\lambda \Lambda_{*}\right] \exp \left[-\frac{\Lambda_{*}^{2}}{4}\right] .
$$

To compute $\operatorname{Pr}[W=L]$ from (3.37), we note that replacing $L$ by $L-1$ corresponds to replacing $\Lambda_{*}$ by $\Lambda_{*}-1 / \sqrt{\rho}$ and, thus, backward differencing the negative of (3.37) asymptotically corresponds to multiplying by $\exp [\lambda / \sqrt{\rho}]-1 \sim \lambda / \sqrt{\rho}$. Also, $\lambda^{2}=2 \log \log \rho$ and, hence, $\lambda / \sqrt{\rho}$ times (3.37) is the same as the expression in (3.38), so that (2.9) and (2.10) indeed match.

We examine very large values of $L$, which have $L \gg O(\rho)$. The argument leading to (3.19) assumed that the summand in (3.15) had an interior maximum at $j=\rho \zeta_{*}$, but $\zeta_{*} \rightarrow 0$ (with $\zeta_{*}=O(1 / \nu)$ ) as $v \rightarrow \infty$. We re-examine (3.15) with the scaling $L=\rho^{2} \tau=O\left(\rho^{2}\right)$. Approximating the summand in (3.15) for $L / \rho \rightarrow \infty$ and $j / L \rightarrow 0$ and again using Stirling's formula in reverse leads to

$$
\operatorname{Pr}[W>L] \sim \frac{\rho^{L}}{L(L !)}\left(\sum_{j=1}^{\infty} \frac{\mathrm{e}^{-2 \rho}}{(j-1) !} \frac{1}{\tau^{j}}\right) .
$$

Here we also used $(L+j) ! \sim L ! L^{j}$ and $\rho^{2} / L=1 / \tau$. Note that (3.39) is similar to (3.16), except that the factor $\zeta_{*}$ in (3.16) must be replaced by $j / \rho$ and taken inside the summation. For $\zeta_{*} \rightarrow 0$, we have $\left(1-\zeta_{*}\right)\left(v+\zeta_{*}-1\right) \sim v=L / \rho$. Evaluating the sum in (3.39) we conclude that, for $L=O\left(\rho^{2}\right)$,

$$
\operatorname{Pr}[W>L] \sim \frac{\rho^{L}}{(\tau L) L !} \mathrm{e}^{-2 \rho} \mathrm{e}^{1 / \tau},
$$

where $L$ ! can be further approximated by the Stirling formula. But, for $v \rightarrow \infty$, we have

$$
\begin{aligned}
\mathrm{e}^{\rho \phi(v)} & =\exp \left[\rho\left(-v \log v+v-2+\frac{1}{v}+O\left(\frac{1}{v^{2}}\right)\right)\right] \\
& \sim\left(\frac{\rho}{L}\right)^{L} \mathrm{e}^{L} \mathrm{e}^{-2 \rho} \exp \left[\frac{\rho^{2}}{L}\right]
\end{aligned}
$$

and $C-1 \sim 2 / v^{2}$ with which

$$
f(v) \sim \frac{1}{\sqrt{2 \pi \rho}} v^{-5 / 2} .
$$

Combining (3.41) and (3.42) in (3.19) we obtain (3.40), with $L$ ! approximated by Stirling's formula. This shows that (3.19), and hence (2.11), remains valid for $L=O\left(\rho^{2}\right)$ (i.e. $v=$ $O(\rho)$ ). In fact, it remains true for arbitrarily large $L$, since then $\operatorname{Pr}[W>L]$ is asymptotically given by the $j=1$ term in the sum in (3.39).

Finally, consider $\rho$ fixed and $L \rightarrow \infty$. We have $\pi(0,0 ; L)=\mathrm{e}^{-\rho}$ and, for $j \geq 1$, the results in [7] show that

$$
\frac{\rho^{j} \mathrm{e}^{-\rho}}{j !}-\pi(j, 0 ; L+j) \sim \frac{1}{(j-1) !} \frac{\mathrm{e}^{-2 \rho}}{L} \frac{\rho^{L+2 j}}{(L+j) !} .
$$


Using (3.43) in (2.6), we see that the term with $j=1$ is asymptotically dominant and we obtain

$$
\operatorname{Pr}[W>L] \sim \frac{\mathrm{e}^{-2 \rho}}{L} \frac{\rho^{L+2}}{(L+1) !}, \quad L \rightarrow \infty, \rho=O(1) .
$$

But the above is equivalent to (2.13), as now

$$
\operatorname{Pr}[W>L] \sim \operatorname{Pr}[W=L+1]
$$

This completes the analysis.

\section{Numerical results}

In this section we compare the asymptotic results from Theorem 2.1 with the exact values. To obtain the exact value of $\operatorname{Pr}[W=L]$, we use the sum for $G_{k}(z)$ in (2.2) to rewrite (2.1) as

$$
\begin{aligned}
& \pi(j, 0 ; L+j)=\frac{(L+j) !}{j !} \rho^{-L} \frac{1}{2 \pi \mathrm{i}} \\
& \times \int_{B r_{+}} \rho^{-z} \Gamma(z) \sum_{l=0}^{j}\left(\begin{array}{l}
j \\
l
\end{array}\right) \rho^{-l} \frac{\Gamma(l-z)}{\Gamma(-z)} \\
& \times\left(\sum_{l=0}^{L+j}\left(\begin{array}{c}
L+j \\
l
\end{array}\right) \rho^{-l} \frac{\Gamma(l-z)}{\Gamma(-z)}\right. \\
& \left.\times \sum_{l=0}^{L+j}\left(\begin{array}{c}
L+j \\
l
\end{array}\right) \rho^{-l} \frac{\Gamma(l-z+1)}{\Gamma(-z+1)}\right)^{-1} \mathrm{~d} z .
\end{aligned}
$$

We can close $B r_{+}$in the left-hand half-plane where the gamma function has singularities at $z=0,-1,-2,-3, \ldots$ and write the integral as a residue series:

$$
\begin{aligned}
\pi(j, 0 ; L+j)= & \frac{(L+j) !}{j !} \rho^{-L} \\
& \times \sum_{n=0}^{\infty}(-\rho)^{n} \frac{\sum_{l=0}^{j}\left(\begin{array}{l}
j \\
l
\end{array}\right) \rho^{-l}(l+n-1) !}{\sum_{l=0}^{L+j}\left(\begin{array}{c}
L+j \\
l
\end{array}\right) \rho^{-l}(l+n-1) ! \sum_{l=0}^{L+j}\left(\begin{array}{c}
L+j \\
l
\end{array}\right) \rho^{-l}(l+n) !} .
\end{aligned}
$$

The term with $n=0$ must be interpreted as a limiting case of the terms with $n>0$. Isolating this term leads to

$$
\begin{aligned}
& \pi(j, 0 ; L+j) \\
& =\frac{(L+j) !}{j !} \rho^{-L} \\
& \quad \times\left(\left(\sum_{l=0}^{L+j}\left(\begin{array}{c}
L+j \\
l
\end{array}\right) \rho^{-l} l !\right)^{-1}\right. \\
& \left.\quad+\sum_{n=1}^{\infty}(-\rho)^{n} \frac{\sum_{l=0}^{j}\left(\begin{array}{l}
j \\
l
\end{array}\right) \rho^{-l}(l+n-1) !}{\sum_{l=0}^{L+j}\left(\begin{array}{c}
L+j \\
l
\end{array}\right) \rho^{-l}(l+n-1) ! \sum_{l=0}^{L+j}\left(\begin{array}{c}
L+j \\
l
\end{array}\right) \rho^{-l}(l+n) !}\right) .
\end{aligned}
$$


We used MAPLE ${ }^{\circledR}$ to evaluate the sum in (4.1). The sum is an alternating sum and the calculation must be done using many digits of precision, especially for larger values of $\rho$. We used (4.1) in (2.5) and summed over $j$ to obtain $\operatorname{Pr}[W \leq L]$, and then computed $\operatorname{Pr}[W=L]$ from $\operatorname{Pr}[W \leq L]-\operatorname{Pr}[W \leq L-1]$.

We denote the asymptotic results in (2.8), (2.9), (2.10), and (2.11) by

$$
\begin{gathered}
L=O(\lambda \sqrt{\rho}), \quad L \sim 2 \lambda \sqrt{\rho}, \\
L=O\left(\rho^{2 / 3}\right), \quad \text { and } \quad L=O(\rho),
\end{gathered}
$$

respectively. Tables 1-4 display the numerical and asymptotic results for $\rho=40,60,80$, and 100 , respectively. We see that, for small $L, L=O(\lambda \sqrt{\rho})$ gives the best approximation, that there is a middle range of $L$, where $L=O\left(\rho^{2 / 3}\right)$ is optimal, and that, for sufficiently large $L$, $L=O(\rho)$ gives the best approximation.

\begin{tabular}{|c|c|c|c|c|c|}
\hline$L$ & Exact value & $L=O(\lambda \sqrt{\rho})$ & $L \sim 2 \lambda \sqrt{\rho}$ & $L=O\left(\rho^{2 / 3}\right)$ & $L=O(\rho)$ \\
\hline 0 & $6.533 \times 10^{-2}$ & $5.306 \times 10^{-2}$ & $2.396 \times 10^{-3}$ & - & - \\
\hline 1 & $7.124 \times 10^{-2}$ & $5.828 \times 10^{-2}$ & $3.176 \times 10^{-3}$ & $2.460 \times 10^{-3}$ & - \\
\hline 2 & $7.522 \times 10^{-2}$ & $6.285 \times 10^{-2}$ & $4.112 \times 10^{-3}$ & $7.401 \times 10^{-3}$ & - \\
\hline 3 & $7.723 \times 10^{-2}$ & $6.658 \times 10^{-2}$ & $5.200 \times 10^{-3}$ & $1.328 \times 10^{-2}$ & - \\
\hline 4 & $7.732 \times 10^{-2}$ & $6.931 \times 10^{-2}$ & $6.422 \times 10^{-3}$ & $1.917 \times 10^{-2}$ & $7.870 \times 10^{-1}$ \\
\hline 5 & $7.566 \times 10^{-2}$ & $7.094 \times 10^{-2}$ & $7.748 \times 10^{-3}$ & $2.445 \times 10^{-2}$ & $5.914 \times 10^{-1}$ \\
\hline 6 & $7.249 \times 10^{-2}$ & $7.145 \times 10^{-2}$ & $9.131 \times 10^{-3}$ & $2.870 \times 10^{-2}$ & $4.571 \times 10^{-1}$ \\
\hline 7 & $6.809 \times 10^{-2}$ & $7.088 \times 10^{-2}$ & $1.051 \times 10^{-2}$ & $3.170 \times 10^{-2}$ & $3.590 \times 10^{-1}$ \\
\hline 8 & $6.279 \times 10^{-2}$ & $6.931 \times 10^{-2}$ & $1.183 \times 10^{-2}$ & $3.337 \times 10^{-2}$ & $2.842 \times 10^{-1}$ \\
\hline 9 & $5.689 \times 10^{-2}$ & $6.689 \times 10^{-2}$ & $1.301 \times 10^{-2}$ & $3.378 \times 10^{-2}$ & $2.257 \times 10^{-1}$ \\
\hline 10 & $5.070 \times 10^{-2}$ & $6.382 \times 10^{-2}$ & $1.398 \times 10^{-2}$ & $3.306 \times 10^{-2}$ & $1.793 \times 10^{-1}$ \\
\hline 11 & $4.445 \times 10^{-2}$ & $6.030 \times 10^{-2}$ & $1.469 \times 10^{-2}$ & $3.141 \times 10^{-2}$ & $1.420 \times 10^{-1}$ \\
\hline 12 & $3.838 \times 10^{-2}$ & $5.656 \times 10^{-2}$ & $1.508 \times 10^{-2}$ & $2.906 \times 10^{-2}$ & $1.121 \times 10^{-1}$ \\
\hline 13 & $3.265 \times 10^{-2}$ & $5.285 \times 10^{-2}$ & $1.515 \times 10^{-2}$ & $2.625 \times 10^{-2}$ & $8.795 \times 10^{-2}$ \\
\hline 15 & $2.262 \times 10^{-2}$ & $4.655 \times 10^{-2}$ & $1.430 \times 10^{-2}$ & $2.007 \times 10^{-2}$ & $5.310 \times 10^{-2}$ \\
\hline 20 & $7.120 \times 10^{-3}$ & - & $8.471 \times 10^{-3}$ & $7.392 \times 10^{-3}$ & $1.301 \times 10^{-2}$ \\
\hline 25 & $1.624 \times 10^{-3}$ & - & $3.003 \times 10^{-3}$ & $1.821 \times 10^{-3}$ & $2.510 \times 10^{-3}$ \\
\hline 30 & $2.720 \times 10^{-4}$ & - & $6.642 \times 10^{-4}$ & $3.138 \times 10^{-4}$ & $3.743 \times 10^{-4}$ \\
\hline 35 & $3.379 \times 10^{-5}$ & - & $9.635 \times 10^{-5}$ & $3.867 \times 10^{-5}$ & $4.286 \times 10^{-5}$ \\
\hline 40 & $3.142 \times 10^{-6}$ & - & $9.611 \times 10^{-6}$ & $3.451 \times 10^{-6}$ & $3.763 \times 10^{-6}$ \\
\hline 45 & $2.207 \times 10^{-7}$ & - & $6.825 \times 10^{-7}$ & $2.245 \times 10^{-7}$ & $2.539 \times 10^{-7}$ \\
\hline 50 & $1.182 \times 10^{-8}$ & - & $3.515 \times 10^{-8}$ & $1.068 \times 10^{-8}$ & $1.321 \times 10^{-8}$ \\
\hline 51 & $6.376 \times 10^{-9}$ & - & $1.870 \times 10^{-8}$ & $5.595 \times 10^{-9}$ & $7.096 \times 10^{-9}$ \\
\hline 52 & $3.404 \times 10^{-9}$ & - & $9.824 \times 10^{-9}$ & $2.895 \times 10^{-9}$ & $3.772 \times 10^{-9}$ \\
\hline 60 & $1.557 \times 10^{-11}$ & - & $3.634 \times 10^{-11}$ & $9.526 \times 10^{-12}$ & $1.679 \times 10^{-11}$ \\
\hline 70 & $7.661 \times 10^{-15}$ & - & $1.076 \times 10^{-14}$ & $2.464 \times 10^{-15}$ & $8.092 \times 10^{-15}$ \\
\hline 80 & $1.495 \times 10^{-18}$ & - & $9.124 \times 10^{-19}$ & $1.850 \times 10^{-19}$ & $1.559 \times 10^{-18}$ \\
\hline 90 & $1.223 \times 10^{-22}$ & - & $2.217 \times 10^{-23}$ & $4.024 \times 10^{-24}$ & $1.264 \times 10^{-22}$ \\
\hline 100 & $4.414 \times 10^{-27}$ & - & $1.544 \times 10^{-28}$ & $2.533 \times 10^{-29}$ & $4.537 \times 10^{-27}$ \\
\hline
\end{tabular}

TABLE 1: Numerical and asymptotic results for $\rho=40$. 
In Table 5 we show where the transitions occur for the various $\rho$. For each $\rho$, we give the value of

$$
\lambda \sqrt{\rho}=\sqrt{\rho} \sqrt{2 \log \log \rho}
$$

and indicate the range of $L$ where a given asymptotic approximation is optimal. Note that the approximation in (2.9), where $L \sim 2 \lambda \sqrt{\rho}$, is never optimal. For each $\rho$, the first transition

TABLE 2: Numerical and asymptotic results for $\rho=60$.

\begin{tabular}{|c|c|c|c|c|c|}
\hline$L$ & Exact value & $L=O(\lambda \sqrt{\rho})$ & $L \sim 2 \lambda \sqrt{\rho}$ & $L=O\left(\rho^{2 / 3}\right)$ & $L=O(\rho)$ \\
\hline 0 & $4.812 \times 10^{-2}$ & $3.756 \times 10^{-2}$ & $1.847 \times 10^{-3}$ & - & - \\
\hline 1 & $5.253 \times 10^{-2}$ & $4.125 \times 10^{-2}$ & $2.346 \times 10^{-3}$ & $1.464 \times 10^{-3}$ & - \\
\hline 2 & $5.607 \times 10^{-2}$ & $4.473 \times 10^{-2}$ & $2.933 \times 10^{-3}$ & $4.545 \times 10^{-3}$ & - \\
\hline 3 & $5.867 \times 10^{-2}$ & $4.791 \times 10^{-2}$ & $3.611 \times 10^{-3}$ & $8.421 \times 10^{-3}$ & - \\
\hline 4 & $6.028 \times 10^{-2}$ & $5.070 \times 10^{-2}$ & $4.375 \times 10^{-3}$ & $1.260 \times 10^{-2}$ & - \\
\hline 5 & $6.092 \times 10^{-2}$ & $5.300 \times 10^{-2}$ & $5.218 \times 10^{-3}$ & $1.671 \times 10^{-2}$ & $7.712 \times 10^{-1}$ \\
\hline 6 & $6.061 \times 10^{-2}$ & $5.477 \times 10^{-2}$ & $6.126 \times 10^{-3}$ & $2.048 \times 10^{-2}$ & $6.113 \times 10^{-1}$ \\
\hline 7 & $5.944 \times 10^{-2}$ & $5.596 \times 10^{-2}$ & $7.082 \times 10^{-3}$ & $2.371 \times 10^{-2}$ & $4.942 \times 10^{-1}$ \\
\hline 8 & $5.749 \times 10^{-2}$ & $5.656 \times 10^{-2}$ & $8.060 \times 10^{-3}$ & $2.628 \times 10^{-2}$ & $4.045 \times 10^{-1}$ \\
\hline 9 & $5.488 \times 10^{-2}$ & $5.828 \times 10^{-2}$ & $9.031 \times 10^{-3}$ & $2.810 \times 10^{-2}$ & $3.336 \times 10^{-1}$ \\
\hline 10 & $5.174 \times 10^{-2}$ & $5.600 \times 10^{-2}$ & $9.965 \times 10^{-3}$ & $2.918 \times 10^{-2}$ & $2.762 \times 10^{-1}$ \\
\hline 11 & $4.820 \times 10^{-2}$ & $5.493 \times 10^{-2}$ & $1.083 \times 10^{-2}$ & $2.954 \times 10^{-2}$ & $2.291 \times 10^{-1}$ \\
\hline 12 & $4.438 \times 10^{-2}$ & $5.341 \times 10^{-2}$ & $1.159 \times 10^{-2}$ & $2.923 \times 10^{-2}$ & $1.900 \times 10^{-1}$ \\
\hline 13 & $4.041 \times 10^{-2}$ & $5.152 \times 10^{-2}$ & $1.221 \times 10^{-2}$ & $2.834 \times 10^{-2}$ & $1.574 \times 10^{-1}$ \\
\hline 14 & $3.640 \times 10^{-2}$ & $4.935 \times 10^{-2}$ & $1.267 \times 10^{-2}$ & $2.699 \times 10^{-2}$ & $1.300 \times 10^{-1}$ \\
\hline 15 & $3.244 \times 10^{-2}$ & $4.700 \times 10^{-2}$ & $1.295 \times 10^{-2}$ & $2.526 \times 10^{-2}$ & $1.071 \times 10^{-1}$ \\
\hline 16 & $2.862 \times 10^{-2}$ & $4.459 \times 10^{-2}$ & $1.305 \times 10^{-2}$ & $2.328 \times 10^{-2}$ & $8.790 \times 10^{-2}$ \\
\hline 20 & $1.569 \times 10^{-2}$ & $5.828 \times 10^{-2}$ & $1.158 \times 10^{-2}$ & $1.458 \times 10^{-2}$ & $3.800 \times 10^{-2}$ \\
\hline 25 & $5.995 \times 10^{-3}$ & $6.994 \times 10^{-2}$ & $7.201 \times 10^{-3}$ & $6.170 \times 10^{-3}$ & $1.171 \times 10^{-2}$ \\
\hline 30 & $1.833 \times 10^{-3}$ & - & $3.174 \times 10^{-3}$ & $2.000 \times 10^{-3}$ & $3.063 \times 10^{-3}$ \\
\hline 35 & $4.527 \times 10^{-4}$ & - & $1.013 \times 10^{-3}$ & $5.087 \times 10^{-4}$ & $6.733 \times 10^{-4}$ \\
\hline 40 & $9.073 \times 10^{-5}$ & - & $2.405 \times 10^{-4}$ & $1.029 \times 10^{-4}$ & $1.237 \times 10^{-4}$ \\
\hline 45 & $1.484 \times 10^{-5}$ & - & $4.363 \times 10^{-5}$ & $1.670 \times 10^{-5}$ & $1.895 \times 10^{-5}$ \\
\hline 50 & $1.989 \times 10^{-6}$ & - & $6.201 \times 10^{-6}$ & $2.186 \times 10^{-6}$ & $2.417 \times 10^{-6}$ \\
\hline 55 & $2.195 \times 10^{-7}$ & - & $7.029 \times 10^{-7}$ & $2.315 \times 10^{-7}$ & $2.571 \times 10^{-7}$ \\
\hline 60 & $2.003 \times 10^{-8}$ & - & $6.423 \times 10^{-8}$ & $1.989 \times 10^{-8}$ & $2.281 \times 10^{-8}$ \\
\hline 65 & $1.519 \times 10^{-9}$ & - & $4.755 \times 10^{-9}$ & $1.388 \times 10^{-9}$ & $1.692 \times 10^{-9}$ \\
\hline 66 & $8.869 \times 10^{-10}$ & - & $2.755 \times 10^{-9}$ & $7.946 \times 10^{-10}$ & $9.847 \times 10^{-10}$ \\
\hline 67 & $5.142 \times 10^{-10}$ & - & $1.583 \times 10^{-9}$ & $4.513 \times 10^{-10}$ & $5.689 \times 10^{-10}$ \\
\hline 68 & $2.960 \times 10^{-10}$ & - & $9.019 \times 10^{-10}$ & $2.542 \times 10^{-10}$ & $3.264 \times 10^{-10}$ \\
\hline 70 & $9.597 \times 10^{-11}$ & - & $2.856 \times 10^{-10}$ & $7.868 \times 10^{-11}$ & $1.052 \times 10^{-10}$ \\
\hline 80 & $2.254 \times 10^{-13}$ & - & $5.513 \times 10^{-13}$ & $1.360 \times 10^{-13}$ & $2.412 \times 10^{-13}$ \\
\hline 90 & $2.669 \times 10^{-16}$ & - & $4.626 \times 10^{-16}$ & $1.029 \times 10^{-16}$ & $2.812 \times 10^{-16}$ \\
\hline 100 & $1.637 \times 10^{-19}$ & - & $1.687 \times 10^{-19}$ & $3.411 \times 10^{-20}$ & $1.706 \times 10^{-19}$ \\
\hline 110 & $5.336 \times 10^{-23}$ & - & $2.673 \times 10^{-23}$ & $4.947 \times 10^{-24}$ & $5.521 \times 10^{-23}$ \\
\hline 120 & $9.484 \times 10^{-27}$ & - & $1.840 \times 10^{-27}$ & $3.138 \times 10^{-28}$ & $9.758 \times 10^{-27}$ \\
\hline 130 & $9.416 \times 10^{-31}$ & - & $5.508 \times 10^{-32}$ & $8.698 \times 10^{-33}$ & $9.647 \times 10^{-31}$ \\
\hline 140 & $5.346 \times 10^{-35}$ & - & $7.165 \times 10^{-37}$ & $1.053 \times 10^{-37}$ & $5.459 \times 10^{-35}$ \\
\hline 150 & $2.000 \times 10^{-39}$ & - & $4.050 \times 10^{-42}$ & $5.567 \times 10^{-43}$ & $1.808 \times 10^{-39}$ \\
\hline
\end{tabular}


TABLE 3: Numerical and asymptotic results for $\rho=80$.

\begin{tabular}{|c|c|c|c|c|c|}
\hline$L$ & Exact value & $L=O(\lambda \sqrt{\rho})$ & $L \sim 2 \lambda \sqrt{\rho}$ & $L=O\left(\rho^{2 / 3}\right)$ & $L=O(\rho)$ \\
\hline 0 & $3.887 \times 10^{-2}$ & $2.968 \times 10^{-2}$ & $1.537 \times 10^{-3}$ & - & - \\
\hline 1 & $4.232 \times 10^{-2}$ & $3.249 \times 10^{-2}$ & $1.901 \times 10^{-3}$ & $1.009 \times 10^{-3}$ & - \\
\hline 2 & $4.530 \times 10^{-2}$ & $3.521 \times 10^{-2}$ & $2.322 \times 10^{-3}$ & $3.188 \times 10^{-3}$ & - \\
\hline 3 & $4.773 \times 10^{-2}$ & $3.780 \times 10^{-2}$ & $2.804 \times 10^{-3}$ & $6.015 \times 10^{-3}$ & - \\
\hline 4 & $4.958 \times 10^{-2}$ & $4.021 \times 10^{-2}$ & $3.345 \times 10^{-3}$ & $9.175 \times 10^{-3}$ & - \\
\hline 5 & $5.083 \times 10^{-2}$ & $4.237 \times 10^{-2}$ & $3.945 \times 10^{-3}$ & $1.243 \times 10^{-2}$ & $9.188 \times 10^{-1}$ \\
\hline 6 & $5.148 \times 10^{-2}$ & $4.425 \times 10^{-2}$ & $4.597 \times 10^{-3}$ & $1.559 \times 10^{-2}$ & $7.375 \times 10^{-1}$ \\
\hline 7 & $5.152 \times 10^{-2}$ & $4.580 \times 10^{-2}$ & $5.294 \times 10^{-3}$ & $1.850 \times 10^{-2}$ & $6.050 \times 10^{-1}$ \\
\hline 8 & $5.101 \times 10^{-2}$ & $4.699 \times 10^{-2}$ & $6.025 \times 10^{-3}$ & $2.106 \times 10^{-2}$ & $5.036 \times 10^{-1}$ \\
\hline 9 & $4.996 \times 10^{-2}$ & $4.781 \times 10^{-2}$ & $6.779 \times 10^{-3}$ & $2.319 \times 10^{-2}$ & $4.231 \times 10^{-1}$ \\
\hline 10 & $4.845 \times 10^{-2}$ & $4.824 \times 10^{-2}$ & $7.537 \times 10^{-3}$ & $2.484 \times 10^{-2}$ & $3.577 \times 10^{-1}$ \\
\hline 11 & $4.654 \times 10^{-2}$ & $4.829 \times 10^{-2}$ & $8.284 \times 10^{-3}$ & $2.598 \times 10^{-2}$ & $3.036 \times 10^{-1}$ \\
\hline 12 & $4.428 \times 10^{-2}$ & $4.798 \times 10^{-2}$ & $9.000 \times 10^{-3}$ & $2.663 \times 10^{-2}$ & $2.582 \times 10^{-1}$ \\
\hline 13 & $4.175 \times 10^{-2}$ & $4.732 \times 10^{-2}$ & $9.665 \times 10^{-3}$ & $2.680 \times 10^{-2}$ & $2.197 \times 10^{-1}$ \\
\hline 14 & $3.903 \times 10^{-2}$ & $4.636 \times 10^{-2}$ & $1.026 \times 10^{-2}$ & $2.653 \times 10^{-2}$ & $1.869 \times 10^{-1}$ \\
\hline 15 & $3.617 \times 10^{-2}$ & $4.513 \times 10^{-2}$ & $1.077 \times 10^{-2}$ & $2.588 \times 10^{-2}$ & $1.588 \times 10^{-1}$ \\
\hline 16 & $3.324 \times 10^{-2}$ & $4.367 \times 10^{-2}$ & $1.117 \times 10^{-2}$ & $2.489 \times 10^{-2}$ & $1.347 \times 10^{-1}$ \\
\hline 17 & $3.030 \times 10^{-2}$ & $4.205 \times 10^{-2}$ & $1.146 \times 10^{-2}$ & $2.364 \times 10^{-2}$ & $1.140 \times 10^{-1}$ \\
\hline 18 & $2.740 \times 10^{-2}$ & $4.032 \times 10^{-2}$ & $1.163 \times 10^{-2}$ & $2.218 \times 10^{-2}$ & $9.624 \times 10^{-2}$ \\
\hline 20 & $2.189 \times 10^{-2}$ & $3.675 \times 10^{-2}$ & $1.157 \times 10^{-2}$ & $1.888 \times 10^{-2}$ & $6.790 \times 10^{-2}$ \\
\hline 25 & $1.097 \times 10^{-2}$ & $3.041 \times 10^{-2}$ & $9.403 \times 10^{-3}$ & $1.059 \times 10^{-2}$ & $2.651 \times 10^{-2}$ \\
\hline 30 & $4.602 \times 10^{-3}$ & $8.445 \times 10^{-2}$ & $5.841 \times 10^{-3}$ & $4.765 \times 10^{-3}$ & $9.238 \times 10^{-3}$ \\
\hline 35 & $1.630 \times 10^{-3}$ & - & $2.805 \times 10^{-3}$ & $1.762 \times 10^{-3}$ & $2.840 \times 10^{-3}$ \\
\hline 40 & $4.902 \times 10^{-4}$ & - & $1.056 \times 10^{-3}$ & $5.430 \times 10^{-4}$ & $7.648 \times 10^{-4}$ \\
\hline 45 & $1.256 \times 10^{-4}$ & - & $3.172 \times 10^{-4}$ & $1.407 \times 10^{-4}$ & $1.798 \times 10^{-4}$ \\
\hline 50 & $2.752 \times 10^{-5}$ & - & $7.734 \times 10^{-5}$ & $3.084 \times 10^{-5}$ & $3.682 \times 10^{-5}$ \\
\hline 55 & $5.170 \times 10^{-6}$ & - & $1.558 \times 10^{-5}$ & $5.741 \times 10^{-6}$ & $6.562 \times 10^{-6}$ \\
\hline 60 & $8.352 \times 10^{-7}$ & - & $2.629 \times 10^{-6}$ & $9.102 \times 10^{-7}$ & $1.017 \times 10^{-6}$ \\
\hline 65 & $1.163 \times 10^{-7}$ & - & $3.754 \times 10^{-7}$ & $1.232 \times 10^{-7}$ & $1.371 \times 10^{-7}$ \\
\hline 70 & $1.401 \times 10^{-8}$ & - & $4.566 \times 10^{-8}$ & $1.424 \times 10^{-8}$ & $1.609 \times 10^{-8}$ \\
\hline 75 & $1.462 \times 10^{-9}$ & - & $4.741 \times 10^{-9}$ & $1.407 \times 10^{-9}$ & $1.646 \times 10^{-9}$ \\
\hline 80 & $1.325 \times 10^{-10}$ & - & $4.209 \times 10^{-10}$ & $1.190 \times 10^{-10}$ & $1.467 \times 10^{-10}$ \\
\hline 81 & $8.063 \times 10^{-11}$ & - & $2.545 \times 10^{-10}$ & $7.130 \times 10^{-11}$ & $8.900 \times 10^{-11}$ \\
\hline 82 & $4.879 \times 10^{-11}$ & - & $1.529 \times 10^{-10}$ & $4.243 \times 10^{-11}$ & $5.370 \times 10^{-11}$ \\
\hline 83 & $2.936 \times 10^{-11}$ & - & $9.131 \times 10^{-11}$ & $2.510 \times 10^{-11}$ & $3.222 \times 10^{-11}$ \\
\hline 90 & $7.193 \times 10^{-13}$ & - & $2.075 \times 10^{-12}$ & $5.344 \times 10^{-13}$ & $7.770 \times 10^{-13}$ \\
\hline 100 & $2.276 \times 10^{-15}$ & - & $5.475 \times 10^{-15}$ & $1.290 \times 10^{-15}$ & $2.418 \times 10^{-15}$ \\
\hline 110 & $4.264 \times 10^{-18}$ & - & $7.732 \times 10^{-18}$ & $1.675 \times 10^{-18}$ & $4.476 \times 10^{-18}$ \\
\hline 120 & $4.800 \times 10^{-21}$ & - & $5.845 \times 10^{-21}$ & $1.170 \times 10^{-21}$ & $4.995 \times 10^{-21}$ \\
\hline 130 & $3.295 \times 10^{-24}$ & - & $2.365 \times 10^{-24}$ & $4.396 \times 10^{-25}$ & $3.407 \times 10^{-24}$ \\
\hline 140 & $1.400 \times 10^{-27}$ & - & $5.123 \times 10^{-28}$ & $8.880 \times 10^{-29}$ & $1.440 \times 10^{-27}$ \\
\hline 150 & $3.734 \times 10^{-31}$ & - & $5.939 \times 10^{-32}$ & $9.641 \times 10^{-33}$ & $3.827 \times 10^{-31}$ \\
\hline 160 & $6.340 \times 10^{-35}$ & - & $3.686 \times 10^{-36}$ & $5.623 \times 10^{-37}$ & $6.478 \times 10^{-35}$ \\
\hline 170 & $6.949 \times 10^{-39}$ & - & $1.224 \times 10^{-40}$ & $1.761 \times 10^{-41}$ & $7.082 \times 10^{-39}$ \\
\hline 180 & $4.981 \times 10^{-43}$ & - & $2.176 \times 10^{-45}$ & $2.962 \times 10^{-46}$ & $5.066 \times 10^{-43}$ \\
\hline
\end{tabular}


TABLE 4: Numerical and asymptotic results for $\rho=100$.

\begin{tabular}{|c|c|c|c|c|c|}
\hline$L$ & Exact value & $L=O(\lambda \sqrt{\rho})$ & $L \sim 2 \lambda \sqrt{\rho}$ & $L=O\left(\rho^{2 / 3}\right)$ & $L=O(\rho)$ \\
\hline 0 & $3.299 \times 10^{-2}$ & $2.485 \times 10^{-2}$ & $1.334 \times 10^{-3}$ & - & - \\
\hline 1 & $3.582 \times 10^{-2}$ & $2.709 \times 10^{-2}$ & $1.618 \times 10^{-3}$ & $7.536 \times 10^{-4}$ & - \\
\hline 2 & $3.834 \times 10^{-2}$ & $2.930 \times 10^{-2}$ & $1.944 \times 10^{-3}$ & $2.411 \times 10^{-3}$ & - \\
\hline 3 & $4.052 \times 10^{-2}$ & $3.145 \times 10^{-2}$ & $2.314 \times 10^{-3}$ & $4.605 \times 10^{-3}$ & - \\
\hline 4 & $4.232 \times 10^{-2}$ & $3.351 \times 10^{-2}$ & $2.727 \times 10^{-3}$ & $7.112 \times 10^{-3}$ & - \\
\hline 5 & $4.371 \times 10^{-2}$ & $3.542 \times 10^{-2}$ & $3.184 \times 10^{-3}$ & $9.764 \times 10^{-3}$ & - \\
\hline 6 & $4.469 \times 10^{-2}$ & $3.717 \times 10^{-2}$ & $3.683 \times 10^{-3}$ & $1.242 \times 10^{-2}$ & $8.465 \times 10^{-1}$ \\
\hline 7 & $4.525 \times 10^{-2}$ & $3.872 \times 10^{-2}$ & $4.220 \times 10^{-3}$ & $1.498 \times 10^{-2}$ & $7.006 \times 10^{-1}$ \\
\hline 8 & $4.540 \times 10^{-2}$ & $4.005 \times 10^{-2}$ & $4.790 \times 10^{-3}$ & $1.734 \times 10^{-2}$ & $5.890 \times 10^{-1}$ \\
\hline 9 & $4.514 \times 10^{-2}$ & $4.113 \times 10^{-2}$ & $5.386 \times 10^{-3}$ & $1.944 \times 10^{-2}$ & $5.005 \times 10^{-1}$ \\
\hline 10 & $4.451 \times 10^{-2}$ & $4.196 \times 10^{-2}$ & $5.999 \times 10^{-3}$ & $2.123 \times 10^{-2}$ & $4.284 \times 10^{-1}$ \\
\hline 11 & $4.353 \times 10^{-2}$ & $4.251 \times 10^{-2}$ & $6.620 \times 10^{-3}$ & $2.267 \times 10^{-2}$ & $3.686 \times 10^{-1}$ \\
\hline 12 & $4.224 \times 10^{-2}$ & $4.278 \times 10^{-2}$ & $7.238 \times 10^{-3}$ & $2.374 \times 10^{-2}$ & $3.182 \times 10^{-1}$ \\
\hline 13 & $4.067 \times 10^{-2}$ & $4.279 \times 10^{-2}$ & $7.839 \times 10^{-3}$ & $2.445 \times 10^{-2}$ & $2.752 \times 10^{-1}$ \\
\hline 14 & $3.887 \times 10^{-2}$ & $4.254 \times 10^{-2}$ & $8.413 \times 10^{-3}$ & $2.480 \times 10^{-2}$ & $2.383 \times 10^{-1}$ \\
\hline 15 & $3.689 \times 10^{-2}$ & $4.204 \times 10^{-2}$ & $8.945 \times 10^{-3}$ & $2.480 \times 10^{-2}$ & $2.063 \times 10^{-1}$ \\
\hline 16 & $3.476 \times 10^{-2}$ & $4.132 \times 10^{-2}$ & $9.424 \times 10^{-3}$ & $2.450 \times 10^{-2}$ & $1.786 \times 10^{-1}$ \\
\hline 17 & $3.253 \times 10^{-2}$ & $4.039 \times 10^{-2}$ & $9.838 \times 10^{-3}$ & $2.392 \times 10^{-2}$ & $1.544 \times 10^{-1}$ \\
\hline 18 & $3.024 \times 10^{-2}$ & $3.930 \times 10^{-2}$ & $1.018 \times 10^{-2}$ & $2.310 \times 10^{-2}$ & $1.332 \times 10^{-1}$ \\
\hline 19 & $2.792 \times 10^{-2}$ & $3.808 \times 10^{-2}$ & $1.043 \times 10^{-2}$ & $2.208 \times 10^{-2}$ & $1.148 \times 10^{-1}$ \\
\hline 20 & $2.562 \times 10^{-2}$ & $3.675 \times 10^{-2}$ & $1.060 \times 10^{-2}$ & $2.091 \times 10^{-2}$ & $9.868 \times 10^{-2}$ \\
\hline 21 & $2.335 \times 10^{-2}$ & $3.535 \times 10^{-2}$ & $1.067 \times 10^{-2}$ & $1.961 \times 10^{-2}$ & $8.462 \times 10^{-2}$ \\
\hline 22 & $2.116 \times 10^{-2}$ & $3.392 \times 10^{-2}$ & $1.064 \times 10^{-2}$ & $1.823 \times 10^{-2}$ & $7.237 \times 10^{-2}$ \\
\hline 23 & $1.905 \times 10^{-2}$ & $3.251 \times 10^{-2}$ & $1.052 \times 10^{-2}$ & $1.681 \times 10^{-2}$ & $6.171 \times 10^{-2}$ \\
\hline 24 & $1.705 \times 10^{-2}$ & $3.114 \times 10^{-2}$ & $1.031 \times 10^{-2}$ & $1.538 \times 10^{-2}$ & $5.246 \times 10^{-2}$ \\
\hline 25 & $1.517 \times 10^{-2}$ & $2.986 \times 10^{-2}$ & $1.001 \times 10^{-2}$ & $1.395 \times 10^{-2}$ & $4.445 \times 10^{-2}$ \\
\hline 30 & $7.738 \times 10^{-3}$ & $2.718 \times 10^{-2}$ & $7.592 \times 10^{-3}$ & $7.688 \times 10^{-3}$ & $1.842 \times 10^{-2}$ \\
\hline 35 & $3.428 \times 10^{-3}$ & - & $4.652 \times 10^{-3}$ & $3.580 \times 10^{-3}$ & $6.938 \times 10^{-3}$ \\
\hline 40 & $1.325 \times 10^{-3}$ & - & $2.323 \times 10^{-3}$ & $1.429 \times 10^{-3}$ & $2.359 \times 10^{-3}$ \\
\hline 45 & $4.485 \times 10^{-4}$ & - & $9.560 \times 10^{-4}$ & $4.930 \times 10^{-4}$ & $7.205 \times 10^{-4}$ \\
\hline 50 & $1.328 \times 10^{-4}$ & - & $3.280 \times 10^{-4}$ & $1.481 \times 10^{-4}$ & $1.972 \times 10^{-4}$ \\
\hline 55 & $3.485 \times 10^{-5}$ & - & $9.502 \times 10^{-5}$ & $3.886 \times 10^{-5}$ & $4.831 \times 10^{-5}$ \\
\hline 60 & $8.058 \times 10^{-6}$ & - & $2.355 \times 10^{-5}$ & $8.943 \times 10^{-6}$ & $1.058 \times 10^{-5}$ \\
\hline 65 & $1.646 \times 10^{-6}$ & - & $5.046 \times 10^{-6}$ & $1.808 \times 10^{-6}$ & $2.069 \times 10^{-6}$ \\
\hline 70 & $2.979 \times 10^{-7}$ & - & $9.431 \times 10^{-7}$ & $3.217 \times 10^{-7}$ & $3.613 \times 10^{-7}$ \\
\hline 75 & $4.782 \times 10^{-8}$ & - & $1.546 \times 10^{-7}$ & $5.042 \times 10^{-8}$ & $5.639 \times 10^{-8}$ \\
\hline 80 & $6.822 \times 10^{-9}$ & - & $2.231 \times 10^{-8}$ & $6.968 \times 10^{-9}$ & $7.862 \times 10^{-9}$ \\
\hline 85 & $8.665 \times 10^{-10}$ & - & $2.838 \times 10^{-9}$ & $8.497 \times 10^{-10}$ & $9.799 \times 10^{-10}$ \\
\hline 90 & $9.812 \times 10^{-11}$ & - & $3.185 \times 10^{-10}$ & $9.144 \times 10^{-11}$ & $1.092 \times 10^{-10}$ \\
\hline 93 & $2.515 \times 10^{-11}$ & - & $8.072 \times 10^{-11}$ & $2.261 \times 10^{-11}$ & $2.777 \times 10^{-11}$ \\
\hline 94 & $1.583 \times 10^{-11}$ & - & $5.058 \times 10^{-11}$ & $1.405 \times 10^{-11}$ & $1.744 \times 10^{-11}$ \\
\hline 95 & $9.919 \times 10^{-12}$ & - & $3.153 \times 10^{-11}$ & $8.689 \times 10^{-12}$ & $1.090 \times 10^{-11}$ \\
\hline 96 & $6.188 \times 10^{-12}$ & - & $1.956 \times 10^{-11}$ & $5.346 \times 10^{-12}$ & $6.784 \times 10^{-12}$ \\
\hline 100 & $8.962 \times 10^{-13}$ & - & $2.755 \times 10^{-12}$ & $7.290 \times 10^{-13}$ & $9.743 \times 10^{-13}$ \\
\hline 110 & $5.252 \times 10^{-15}$ & - & $1.446 \times 10^{-14}$ & $3.536 \times 10^{-15}$ & $5.614 \times 10^{-15}$ \\
\hline 120 & $1.994 \times 10^{-17}$ & - & $4.602 \times 10^{-17}$ & $1.044 \times 10^{-17}$ & $2.105 \times 10^{-17}$ \\
\hline 130 & $4.952 \times 10^{-20}$ & - & $8.884 \times 10^{-20}$ & $1.877 \times 10^{-20}$ & $5.180 \times 10^{-20}$ \\
\hline 140 & $8.119 \times 10^{-23}$ & - & $1.040 \times 10^{-22}$ & $2.055 \times 10^{-23}$ & $8.433 \times 10^{-23}$ \\
\hline 150 & $8.874 \times 10^{-26}$ & - & $7.387 \times 10^{-26}$ & $1.369 \times 10^{-26}$ & $9.166 \times 10^{-26}$ \\
\hline 160 & $6.525 \times 10^{-29}$ & - & $3.182 \times 10^{-29}$ & $5.549 \times 10^{-30}$ & $6.711 \times 10^{-29}$ \\
\hline 170 & $3.259 \times 10^{-32}$ & - & $8.313 \times 10^{-33}$ & $1.369 \times 10^{-33}$ & $3.340 \times 10^{-32}$ \\
\hline 180 & $1.116 \times 10^{-35}$ & - & $1.317 \times 10^{-36}$ & $2.053 \times 10^{-37}$ & $1.140 \times 10^{-35}$ \\
\hline 190 & $2.642 \times 10^{-39}$ & - & $1.266 \times 10^{-40}$ & $1.873 \times 10^{-41}$ & $2.693 \times 10^{-39}$ \\
\hline 200 & $4.366 \times 10^{-43}$ & - & $7.380 \times 10^{-45}$ & $1.039 \times 10^{-45}$ & $4.442 \times 10^{-43}$ \\
\hline 210 & $5.080 \times 10^{-47}$ & - & $2.610 \times 10^{-49}$ & $3.504 \times 10^{-50}$ & $5.161 \times 10^{-47}$ \\
\hline 220 & $4.198 \times 10^{-51}$ & - & $5.596 \times 10^{-54}$ & $7.180 \times 10^{-55}$ & $4.259 \times 10^{-51}$ \\
\hline 230 & $2.484 \times 10^{-55}$ & - & $7.279 \times 10^{-59}$ & $8.942 \times 10^{-60}$ & $2.517 \times 10^{-55}$ \\
\hline
\end{tabular}


TABLE 5 .

\begin{tabular}{rcccc}
\hline$\rho$ & $\lambda \sqrt{\rho}$ & $L=O(\lambda \sqrt{\rho})$ & $L=O\left(\rho^{2 / 3}\right)$ & $L=O(\rho)$ \\
\hline 40 & 10.22 & $0 \leq L \leq 10$ & $11 \leq L \leq 50$ & $51 \leq L$ \\
60 & 13.01 & $0 \leq L \leq 13$ & $14 \leq L \leq 66$ & $67 \leq L$ \\
80 & 15.38 & $0 \leq L \leq 15$ & $16 \leq L \leq 80$ & $81 \leq L$ \\
100 & 17.48 & $0 \leq L \leq 18$ & $19 \leq L \leq 93$ & $94 \leq L$ \\
\hline
\end{tabular}

TABLE 6.

\begin{tabular}{rcc}
\hline$\rho$ & $\sqrt{\rho}$ & $L$ where $\operatorname{Pr}[W=L]$ is maximal \\
\hline 40 & 6.32 & 4 \\
60 & 7.75 & 5 \\
80 & 8.94 & 7 \\
100 & 10 & 8 \\
\hline
\end{tabular}

occurs roughly at $\lambda \sqrt{\rho}$. The second transition (from $L=O\left(\rho^{2 / 3}\right)$ to $L=O(\rho)$ ) occurs roughly at $L=\rho$, but the ratio of this transition point to $\rho$ decreases slowly, which is consistent with the fractional power law $L=O\left(\rho^{2 / 3}\right)$.

The reason that $L \sim 2 \lambda \sqrt{\rho}$ is never optimal is as follows. We have $2 \lambda \sqrt{\rho}=\rho^{2 / 3}$ when $\rho \approx 5038$ and $\rho^{2 / 3}$ exceeds $2 \lambda \sqrt{\rho}$ only for $\rho>5038$. Thus, we would not expect to numerically resolve these two scales for the moderate values of $\rho$ considered here. For very large $\rho$, however, it becomes problematic to evaluate the sum in (4.1).

In Table 6 we indicate the value of $L$ where $\operatorname{Pr}[W=L]$ is maximal. We note that this value of $L$ is numerically slightly less than $\sqrt{\rho}$, while the asymptotic results predict that $\operatorname{Pr}[W=L]$ should be largest at or near $\sqrt{\rho} \sqrt{2 \log \log \rho}$. Apparently, $\rho=100$ is not large enough to see the effects of the factor $\sqrt{2 \log \log \rho}$.

Tables 1-4 show that the asymptotic results for the scales $L=O\left(\rho^{2 / 3}\right)$ and $L=O(\rho)$ are reasonably accurate, but those for $L=O(\lambda \sqrt{\rho})$ and $L \sim 2 \lambda \sqrt{\rho}$ lead to much larger errors. For example, Table 4 shows that, for $0 \leq L \leq 18$, the approximation for $L=O(\lambda \sqrt{\rho})$ is in error by about $30 \%$ in the worst case. In contrast, when $L \geq 94$, the approximation for $L=O(\rho)$ has errors of at most $10 \%$, which decreases to about $2 \%$ when $L=200=2 \rho$.

These error trends are consistent with the asymptotic analysis, which showed that, when $L=O(\rho)$, the next order term is smaller than the leading term by a factor of $\rho^{-1}$. However, the expansions on the scales $L=O(\lambda \sqrt{\rho})$ and $L \sim 2 \lambda \sqrt{\rho}$ have error terms that are either $O(1 / \lambda)$ or $O\left(1 / \lambda^{2}\right)$, and are thus smaller than the leading term only by a factor of $1 / \log \log \rho$. We revisit the calculations by noting that (3.2) has the error term

$$
\operatorname{Pr}[W=0]=\frac{1}{\sqrt{\rho}}\left(\int_{-\infty}^{\infty} \exp \left[-\frac{\alpha^{2}}{4}\right] \rho^{-z_{0} / 2} \frac{\Gamma\left(z_{0}+1\right)}{\Delta(\alpha)} \mathrm{d} \alpha\right)\left(1+O_{\mathrm{R}}\left(\frac{1}{\sqrt{\rho}}\right)\right) .
$$

Here $O_{\mathrm{R}}$ means that the error is 'roughly' $O(1 / \sqrt{\rho})$, possibly with some logarithmic factors. It is only upon expanding (4.2) further that we obtain the much larger $(O(1 / \log \log \rho))$ error terms. Similar comments apply to $\operatorname{Pr}[W=L]$ in (3.11). Thus, we would expect that a more accurate approximation to $\operatorname{Pr}[W=0]$ would result by using (4.2) rather than (2.7), but this is of course at the expense of having a much more complicated approximation. 
We can recast the integral in (4.2) as follows. We recall that $z_{0}(\alpha)$ satisfies $D_{z}(-\alpha)=0$, and differentiating this with respect to $\alpha$ yields

$$
\left(\left.\frac{\partial D_{z}(-\alpha)}{\partial z}\right|_{z=z_{0}(\alpha)}\right) z_{0}^{\prime}(\alpha)-D_{z_{0}(\alpha)}^{\prime}(-\alpha)=0 .
$$

Here $D^{\prime}$ is the derivative of the parabolic cylinder function with respect to its argument.

We change variables from $\alpha$ to $\omega=z_{0}(\alpha)$ in (4.2). In view of (4.3) we have

$$
-D_{z_{0}(\alpha)}(-\alpha)=z_{0}^{\prime}(\alpha) \Delta(\alpha)
$$

and then (4.2) becomes

$$
\operatorname{Pr}[W=0] \sim \frac{1}{\sqrt{\rho}} \int_{0}^{\infty} \frac{\Gamma(\omega+1)}{D_{\omega}^{\prime}(-\alpha(\omega))} \mathrm{e}^{-\theta \omega / 2} \exp \left[-\frac{\alpha^{2}(\omega)}{4}\right] \mathrm{d} \omega .
$$

Here $\alpha(\omega)$ is the inverse function of $\omega=z_{0}(\alpha)$, so that $D_{\omega}(-\alpha(\omega))=0$ and $\alpha$ satisfies: $\alpha \rightarrow \infty$ as $\omega \rightarrow 0 ; \alpha \rightarrow-\infty$ as $\omega \rightarrow \infty$; and $\alpha=0$ if $\omega=1$. However, the numerical evaluation of (4.4) is not trivial, as we must first compute $\alpha(\omega)$ numerically and then employ an accurate quadrature scheme that accounts for the concentration of the integrand near $\omega=0$, which occurs since $\theta=\log \rho$ is large.

\section{References}

[1] Aldous, D. (1986). Some interesting processes arising as heavy traffic limits in an $M / M / \infty$ storage process. Stoch. Process. Applic. 22, 291-313.

[2] Coffman, E. G., JR. And Leighton, F. T. (1989). A provably efficient algorithm for dynamic storage allocation. J. Comput. Syst. Sci. 8, 2-35.

[3] Coffman, E. G., JR. And Mitrani, I. (1988). Storage of single server queues. In Queueing Theory and Its Applications (CWI Monogr. 7), eds O. J. Boxma and R. Syski, North-Holland, Amsterdam, pp. 193-205.

[4] Coffman, E. G., JR., Flatto, L. and Leighton, F. T. (1990). First fit allocation of queues: tight probabilistic bounds on wasted space. Stoch. Process. Applic. 36, 311-330.

[5] Coffman, E. G., Jr., Kadota, T. T. and Shepp, L. A. (1985). A stochastic model of fragmentation in dynamic storage allocation. SIAM J. Comput. 14, 416-425.

[6] KNESSL, C. (2000). Asymptotic expansions for a stochastic model of queue storage. Ann. Appl. Prob. 10, 592-615.

[7] KNESSL, C. (2004). Some asymptotic results for the M/M/ $\infty$ queue with ranked servers. Queueing Systems 47, 201-250.

[8] Kosten, L. (1937). Uber Sperrungswahrscheinlichkeiten bei Staffelschaltungen. Electra Nachrichten-Technik 14, 5-12.

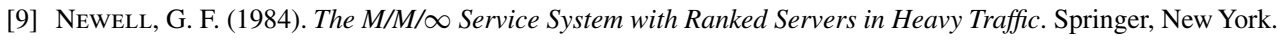

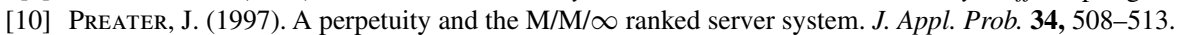

[11] Sohn, E. And Knessl, C. (2008). A simple direct solution to a storage allocation model. Appl. Math. Lett. 21, $172-175$. 